\title{
Axiomatizations of a positional power score and measure for hierarchies
}

\author{
René van den Brink • Frank Steffen
}

Received: 25 November 2008 / Accepted: 21 January 2011 / Published online: 20 February 2011

(C) The Author(s) 2011. This article is published with open access at Springerlink.com

\begin{abstract}
The extant literature on positional power in hierarchies is mainly restricted to the analysis of power in terms of the authority structure. This is based upon the bare positions of actors and ignores the existence of decision-making mechanisms. The few studies, which also take into account decision-making mechanisms, are 'membership-based'. In an earlier paper we demonstrated that such an approach is, in general, inappropriate. As a solution we suggested an 'action-based' approach and developed a power score and measure ascribing positional power to actors in hierarchies. In this paper we axiomatize this power score and measure and illustrate their applicability.
\end{abstract}

Keywords Hierarchies · Decision-making mechanism - Power - Positional power · Power score $\cdot$ Power measure $\cdot$ Binary voting $\cdot$ Axiomatization

JEL Classification C79 $\cdot$ D02 $\cdot$ D71

\section{Introduction}

Power is a core concept in the analysis and design of organizations. Positional power results from the interplay of two components of an organization's architecture: the arrangement of positions in the organization and the decision-making mechanism in use. One of the problems with the extant literature on positional power in hierarchies is that it is mainly restricted

\footnotetext{
${ }^{1}$ See, for instance, Copeland (1951), Russett (1968), Grofman and Owen (1982), Daudi (1986), Brams (1968), van den Brink (1994, 2002), van den Brink and Gilles (2000), Mizruchi and Potts (1998), Hu and Shapley (2003), Herings et al. (2005), and the references therein.
}

R. van den Brink $(\bowtie)$

Department of Econometrics and Tinbergen Institute, VU University, De Boelelaan 1105, 1081 HV Amsterdam, The Netherlands

e-mail: jrbrink@feweb.vu.nl

F. Steffen

Management School, The University of Liverpool, Chatham Street, Liverpool L69 7ZH, UK

e-mail: steffen2@liv.ac.uk 
to the analysis of power in terms of the bare positions of the actors. ${ }^{1}$ While such an analysis informs us about the authority structure within an organization, it ignores the existence of decision-making mechanisms completely. The few studies which take into account the decision-making mechanism make all use of adaptations of well-established approaches for the analysis of power in non-hierarchical organizations such as the Banzhaf (1965) measure. Hence, they are all based on the structure of a simple game, i.e., they are 'membershipbased'. In van den Brink and Steffen (2008) we demonstrated that such an approach is, in general, inappropriate for characterizing power in hierarchies. As a solution we suggested an action-based approach represented by an extensive game form. Based on this approach we developed a power score and measure that can be applied to ascribe positional power to actors in hierarchies, and sequential decision making mechanisms in general. ${ }^{2}$

Hierarchies form a certain subclass of organizational architectures. Following van den Brink (1994) they distinguish themselves from other organizational architectures by the arrangement of their members being connected via directed relations, which we interpret as dominance (or superior to) relations. Loosely speaking, we can say that an actor $i$ in a dominating position has an influence on the 'powers' of other actors who are in positions that are dominated by $i$. Domination can be either indirect or direct, i.e., with or without intermediate actors. Actors in dominating positions are called superiors (or principals)—bosses or managers in common parlance-while the actors in dominated positions are called subordinates (or agents). If we refer to a superior who directly dominates another actor, the dominating actor is called a predecessor, and if we refer to a subordinate who is directly dominated by another actor, the dominated actor is called a successor. ${ }^{3}$

The second component of an organizational architecture we refer to in our analysis is the decision-making mechanism (DMM) in use. A DMM consists of a decision rule together with a decision-making procedure. A decision-making procedure provides the course of actions of the actors for a collective decision and determines the actions to be counted, i.e., which actions belong to the domain of the decision rule. The sets of actions from which an individual actor can choose are created by the proposals submitted to the organization. The choices within the organization are made by one or more actors where each of these actors has to perform an action to make its individual choice effective. How those actions and the outcomes are linked is given by the decision rule. In other words: a decision rule is a function that maps ordered sets of individually chosen actions into outcomes.

In this paper we propose an axiomatization of the power score and measure for positional power in hierarchies, introduced in van den Brink and Steffen (2008), for one of the most discussed decision-making situations in the voting power literature, namely that of binary voting. ${ }^{4}$ The paper is organized as follows. In Sect. 2 we discuss some preliminaries on collective decision making in hierarchies, power and directed graphs. In Sect. 3 we describe the model and the power score and measure. In Sect. 4 we provide axiomatizations of the power score, while Sect. 5 contains an axiomatization of the power measure. In Sect. 6 we supply a generalized version of our power score and corresponding axiomatizations. Moreover, we demonstrate that the generalization has no effect on our power measure. In Sect. 7 we

\footnotetext{
${ }^{2}$ Note that this approach is more general than Felsenthal and Machover's (1996) roll-call model which assumes that all sequential orders of actors choosing their actions are equally likely and does not allow for the existence of hierarchical relations among the actors.

${ }^{3}$ Note that if we just make use of the term 'domination' without further specification, we allow for indirect and direct domination.

${ }^{4}$ In Laruelle and Valenciano's (2008) parlance this is called a take-it-or-leave-it decision-making situation as it leaves no room for negotiation and bargaining.
} 
give two potential applications. In Sect. 8 we illustrate the construction of the membershipbased approaches to the measurement of positional power in hierarchies and undertake a critical assessment. Finally, in Sect. 9 we indicate the applicability of our approach to other collective decision-making situations such as those in the European Union and conclude with some remarks on the relation of our model to Sah and Stiglitz's $(1985,1985)$ work on hierarchies.

\section{Preliminaries}

\subsection{Collective decision making in hierarchies}

There exist two DMM's for hierarchies, the One Desk Conjunctive and the One Desk Disjunctive DMM which can be regarded to be the elementary, bottom-up, DMMs for hierarchies as they form the root of the other DMMs discussed in the literature. ${ }^{5}$ They are the simplest DMMs that take into account the existence of the dominance structure which distinguishes a hierarchy from any other organizational architecture. Both DMMs are jointly characterized by a set of nine assumptions. These assumptions are:

1. Proposals submitted to the hierarchy are exogenous: it is the task of the hierarchy either to accept or reject the proposal, i.e., we have a binary outcome set: $\{$ acceptance, rejection\}.

2. A proposal can be submitted to the hierarchy only once.

3. A hierarchy contains a finite set of members $N,|N| \geq 2$, whose actions bring about the decision of the hierarchy.

4. Each actor $i \in N$ has a binary action set, $\{y e s, n o\}$, to choose from where yes means that $i$ supports the proposal and no that $i$ rejects the proposal. ${ }^{6}$

5. The direction of the decision-making procedure through the hierarchy is bottom-up.

6. New proposals entering the organization can be received only by bottom actors being actors in positions with no successors.

7. A new proposal can be received only by one bottom actor at the same time (One Desk Model).

8. The choice of the yes-action results (i) in a final approval if actor $i$ is the top actor, i.e., if $i$ has no predecessor, or (ii) in forwarding the proposal to one or more predecessors if $i$ is not the top actor.

9. The choice of the no-action results in a final rejection, if (i) actor $i$ is a bottom actor, or (ii) if for the actor who has forwarded the proposal to $i$ there is no other predecessor left to ask for an approval of the proposal whose individual approval contains the potential of a final approval. If such other predecessor as in case (ii) exists the choice of the no-action results in forwarding the proposal to such an actor.

Assumptions 1-4 are also common in the analysis of power in non-hierarchical organizations. The other assumptions are additional for hierarchies. Both DMM's to which we referred above satisfy assumptions 1-9. They differ with respect to the following two assumptions, where each satisfies either one of these two assumptions.

\footnotetext{
${ }^{5}$ See van den Brink and Steffen (2008) for an overview.

${ }^{6}$ Even being a very common assumption it is quite restrictive for non-hierarchical decision-making situations as this excludes the option to abstain as a tertium-quid which can have a considerable impact on the power distribution among the actors (see, for instance, Felsenthal and Machover 1997; Braham and Steffen 2002; and also Fischer 1999). However, in a hierarchical decision-making situation, it is unusual for actors to be allowed to abstain.
} 
10. (One Desk Conjunctive DMM) For the approval of a new proposal received by bottom actor $i$, the consent of all superiors of $i$ is necessary and sufficient.

11. (One Desk Disjunctive DMM) For the approval of a new proposal received by bottom actor $i$, the consent of all superiors of $i$ along one path up to the top is necessary and sufficient.

These two DMM's respect the following two principles. Both reflect the very basic motivation for the creation of a hierarchy being the idea of delegation, for instance, as a cost reduction device. ${ }^{7}$

P1. Reduction of the breadth of the hierarchy involved in a particular decision by a truncation of the hierarchy.

P2. Permission for non-top actors to make certain types of final decisions on behalf of the whole hierarchy even before one of their superiors has been involved.

The principles ensure that in certain instances particular actors are intentionally excluded from decisions, i.e., proposals are not necessarily presented to all members of a hierarchy. P2, especially, which is implemented by Assumption 9 for the above DMMs, is an essential characteristic of DMMs in hierarchies which is often overlooked in the literature (see Sect. 8). ${ }^{8}$

In this paper we restrict our attention to strict hierarchies that are represented by (rooted) directed trees (see Sect. 2.3 and also, e.g., Radner 1992). For such strict hierarchies the Conjunctive and Disjunctive DMM's coincide and therefore we will consider only the One Desk Conjunctive DMM. Note that, for strict hierarchies, Assumption 9 can be simplified by saying that the choice of the no-action results in a final rejection for any actor who receives the proposal.

\subsection{Power}

Our understanding of 'power' is based on Harré (1970) and Morriss (1987/2002) who define power as a concept that always refers to a generic ability or capacity of an actor in a social context: what the actor is able to do against the actual or possible resistance of at least one other actor. ${ }^{9}$ Following Braham (2008) we say that an actor $i$ has power with respect to a

\footnotetext{
${ }^{7}$ See van den Brink and Steffen (2008), referring to Mackenzie (1976: 103) and Shapley and Palamara (2000b).

${ }^{8}$ Note that both principles which are applied here to create exclusion can also be distinguished by the origin of the exclusion, i.e., whether it lies 'inside' or 'outside' the hierarchy. Applying P1 actors with a contact to the outside world may experience exclusion due to the behavior of the outside world (external exclusion): an actor in a hierarchy who has 'only' the task to receive proposals from the outside world and can either reject them on its own or can pass them on for approval to other actors in the hierarchy may be excluded from the decision-making procedure if there exists at least one other actor with a contact to the outside world as the latter actor may be the one who receives the proposal. Applying P2 all actors that are a member of at least one subset which is jointly able to approve a proposal forwarded by an actor with a contact to the outside world may encounter exclusion due to the decisions of other actors in the hierarchy (internal exclusion): if an actor is a member of a subset which is able to jointly approve a proposal forwarded from an actor with a contact to the outside world, it can happen that either (i) one of the other members of its subset is in a position to bring the decision-making procedure to an end by rejecting the proposal before the actor in question is allowed to make its decision, or (ii) that the actor with the contact to the outside world who has received the proposal first immediately rejects the proposal, or (iii) that there exists another subset not containing the actor in question which has received the proposal and now decides on it.
}

${ }^{9}$ Note that power as an 'ability' implies that it can be exercised at 'will'. Power in this sense has to be distinguished from an ordinary dispositional power which only says what an object can do in virtue of its 
certain outcome if $i$ has an action (or sequence of actions) such that the performance of the action under the stated or implied conditions will result in that outcome despite the actual or possible resistance of at least one other actor. That is, power is a claim concerning what $i$ is able to do against some resistance of others irrespective of the actual occurrence of the resistance. Thus, power is a capacity or potential which exists whether it is exercised or not. In our context, this capacity is based on the positions of the actors in an organizational architecture. The measurement of power involves the following steps:

(i) The identification of the action profiles, i.e., vectors containing individual actions, within the organization that are sufficient for bringing about an outcome.

(ii) The ascription of power to an individual actor in these action profiles by determining whether the actor has an alternative action that if performed will, ceteris paribus, alter the outcome of the collective action.

(iii) The aggregation of the individual power ascriptions of each actor, giving us a bare power score.

(iv) The weighting of the aggregated power ascriptions of each actor yielding a power measure.

We will make use of these four steps in Sect. 3 explaining our power score and measure.

\subsection{Directed graphs}

We end the preliminaries by describing some basic concepts of directed graphs (or digraphs). A digraph is a set of objects, called nodes, joined by directed links, called arcs. We model a hierarchical structure by such a digraph where the nodes represent the actors and the arcs represent the dominance relations between them. Thus, formally, in our case a digraph is an ordered pair $(N, D)$, where $N \subset \mathbb{N}$ is a finite set of actors and $D$ is a set of ordered pairs of $N$ called arcs of the graph, i.e., $D \subset N \times N$ is a binary relation on $N$. We assume the digraph to be irreflexive, i.e., $(i, i) \notin D$ for all $i \in N$. An arc $(i, j) \in D$ is considered to be directed from node $i$ to node $j$ where $i$ is called a predecessor of $j$ and $j$ is called a successor of $i$ in $D$. Given a digraph $(N, D)$ we denote $S_{D}(i)=\{j \in N \mid(i, j) \in D\}$ as the set of successors of $i$, and the set $\left.S_{D}^{-1}(i)=\{j \in N \mid(j, i) \in D)\right\}$ as the set of predecessors of $i$. (We will omit the subscript $D$ if this leads to no confusion.) Further, we denote by $\widehat{S}_{D}(i)$ the successors of $i$ in the transitive closure of $D$, i.e., $j \in \widehat{S}_{D}(i)$ if and only if there exists a sequence of actors $\left(h_{1}, \ldots, h_{t}\right)$ such that $h_{1}=i, h_{k+1} \in S\left(h_{k}\right)$ for all $1 \leq k \leq t-1$, and $h_{t}=j$. The actors in $\widehat{S}_{D}(i)$ are called the subordinates of $i$, and the actors in $\widehat{S}_{D}^{-1}(i)=\left\{j \in N \mid i \in \widehat{S}_{D}(j)\right\}$ are called the superiors of $i$. In this paper we restrict our attention to hierarchies with a tree structure, i.e., we consider acyclic structures with a unique actor at the top having no predecessors and being superior to all other actors, while all other actors have exactly one predecessor. These structures are also called strict hierarchies. Since in this paper we only consider such strict hierarchies we will refer to them just as hierarchies. We denote the top actor by $i_{0}$, i.e., $\left|S^{-1}\left(i_{0}\right)\right|=0$ and $\left|S^{-1}(j)\right|=1$ for all $j \in N \backslash\left\{i_{0}\right\}$. Note that every directed tree has at least one actor that has no successors. We call these actors in the set $K(D)=\left\{i \in N \mid S_{D}(i)=\emptyset\right\}$ bottom actors. For a subset $T \subseteq N$ we denote by $(T, D(T))$ with $D(T)=\{(i, j) \in D \mid\{i, j\} \subseteq T\}$, the sub-digraph restricted to $T$.

nature (Harré 1970; Morriss 1987/2002). Moreover, following Morriss (1987/2002) what we call 'power' refers only to this term in English (and any exact translation into other languages that might exist), but not to any terms in other languages which are close to 'power'. 


\section{The model}

\subsection{Power in sequential voting}

Presuming that only bottom actors may receive a proposal on their desk, we presuppose that only those have contact with the outside world. Based on this let us assume a voting procedure that combines hierarchical and polyarchical features similar to those of Sah and Stiglitz $(1985,1986)$, but in the context of binary voting. ${ }^{10}$

Assume an organization described by a tree $(N, D)$ on the set of actors $N$. As mentioned above, new proposals enter the organization at one desk of a bottom actor, i.e., an actor in the set $K(D)$. Let us first consider the most simple case, namely that of a line structure. Without loss of generality we consider the line given by $D=\{(i, i+1) \mid i \in\{1, \ldots, n-1\}\}$. So, $S(i)=i+1$ for all $i \in\{1, \ldots, n-1\}$ and $S(n)=\emptyset$. We consider this line as a hierarchy in the sense of Sah and Stiglitz (1986), meaning that the approval of all actors in the line is necessary for acceptance. So, in this case the proposal enters the organization at position $n$. If the actor in this position rejects then the proposal is rejected by the organization, and if it accepts then the proposal goes to actor $n-1$. If that actor $n-1$ rejects then the proposal is rejected and if it accepts then it goes to actor $n-2$, and so on until it (possibly) reaches actor 1 . Acceptance or rejection of the proposal by actor 1 leads to acceptance or rejection, respectively, by the organization. Clearly, in this case the proposal is accepted if, and only if, all actors accept it. Note that this also implies that the proposal has reached all actors. In this specific circumstance all actors seem equally powerful. However, that is not so obvious if one of the actors rejects the proposal since by the sequential nature of the decision making process the proposal will not reach all superior actors who, thus, cannot cast their votes. As mentioned before, the established power measures in the literature do not take account of this sequential feature (Principle P2).

Besides the choice whether to take account of the sequential nature of the decision making process, we must make more assumptions in case the tree is not a line. There then exists more than one actor who might receive the proposal from the outside world, and we must make an assumption regarding which bottom actor receives the proposal under the One Desk Model. Applying the principle of insufficient reason of classical probability theory, ${ }^{11}$ we assume that each bottom actor receives the proposal with equal probability. ${ }^{12}$ When a bottom actor receives a proposal a sequential hierarchical decision-making process in the line from this bottom position to the top will start. ${ }^{13}$ Hence, when measuring power we can focus on truncated hierarchies, i.e., we consider a tree as a union of lines.

\footnotetext{
${ }^{10}$ Thus, the 'correct' choice is endogenously determined by the preference profile and social welfare function, and not exogenously given as in Sah and Stiglitz (1985, 1986). For a discussion of the relation of our model to Sah and Stiglitz's work see Sect. 9.

${ }^{11}$ In the absence of any information about the outside world the application of the principle of insufficient reason appears to be legitimate here as we fulfill the condition that we have a finite probability space consisting of finitely many clearly distinguished indivisible 'atomic events' (Felsenthal et al. 2003).

${ }^{12}$ This assumption will be relaxed in Sect. 6 in which we provide a generalized version of our power score and measure allowing for any probability distribution.

${ }^{13}$ Alternatively, and in line with Sah and Stiglitz's $(1985,1986)$ idea of a polyarchy, one may, for instance, apply an All Desk Model stating that the proposal enters at all bottom positions, and that one full hierarchical approval in one of the lines is necessary and sufficient for an approval by the organization (see the concluding remarks in Sect. 9). Another intermediate version would be a Multi Desk Model assuming that a new proposal enters the organization at more than one but less than all desks belonging to bottom actors.
} 


\subsection{Power score and measure}

In van den Brink and Steffen (2008) a power score and measure are proposed for general DMMs in hierarchies. There we argue, in a more general setting, that step (i), i.e., identifying the action profiles, requires a set-up that allows for an action-based representation of a DMM in an organizational architecture. Similar to models of simultaneous voting, for the One Desk Conjunctive DMM in a binary voting model, the score of an actor is determined by its number of swings. In traditional (simultaneous) voting, an actor has a swing in an action profile where the outcome changes if the actor changes its vote, given that the other actors do not change their votes. This works well in a simultaneous voting situation where every action profile consists of $n$ votes, one for each actor. Usually these profiles are represented by simple games, i.e., sets of 'coalitions' whose members are voting yes, while their complements consist of the actors who vote no. Therefore, this is referred to as a membership-based approach. However, in a sequential DMM as considered here, not all actors vote in every action profile. This creates several problems for the power ascription in step (ii), for example, with respect to the ceteris paribus clause. It might be that with a certain action of actor $i$, another actor $j$ will not vote, but when $i$ changes its action, then $j$ gets to vote and has an influence on the outcome. Then $i$ changing its action might lead to a different outcome, but not necessarily (depending on the voting behavior of $j$ and possibly other actors). On one hand we cannot consider this to be a 'full' swing for actor $i$ since it cannot, for sure, change the outcome, given that the other actors vote the same way (because in the original action profile $j$ did not vote). On the other hand, by changing its vote $i$ can create a situation where the outcome might change, so we can still consider this to be some kind of swing. Therefore, we distinguish between strong and weak swings. In our case of a binary outcome set these are defined as follows.

Definition 3.1 Actor $i$ has a strong swing in an action profile, if by changing its action it changes the outcome for sure (i.e., turns a rejection into an acceptance or vice versa).

Definition 3.2 Actor $i$ has a weak swing in an action profile, if by changing its action it allows other outcomes, but also the original outcome is still possible.

We illustrate this with an example.

Example 1 Consider the hierarchy $(N, D)$ with $N=\{1,2\}$ and $D=\{(2,1)\}$. In a simultaneous voting situation we would consider four action (or voting) profiles:

$$
\text { (yes, yes), (yes, no), (no, yes), and (no,no). }
$$

However, in our DMM, if actor 1 votes no then the voting stops and the proposal is rejected. So, we have only three action profiles: (yes, yes), (yes, no), and (no). Actor 2 has a strong swing in the first two profiles: by changing its vote the outcome changes from acceptance to rejection, respectively the other way round. Actor 1 has a strong swing in profile (yes, yes) since changing its vote would change an acceptance into a definite rejection. However, considering the profile (no) which yields rejection, by changing its vote the outcome might still be rejection, but also acceptance is possible, depending on the vote of actor 2 . Therefore we refer to this as a weak swing. 
It is usually said that an actor $i$ has a positive swing if $i$, by switching from a no- to a yesaction, can alter the outcome from rejection to acceptance, and has a negative swing, if by switching from a yes- to a no-action can alter the outcome from acceptance to rejection.

To formally measure power, taking account of the sequential nature of the DMM, van den Brink and Steffen (2008) introduced a power score and measure as follows. ${ }^{14}$ Aggregating the individual power ascriptions for each actor in order to obtain a power score (step (iii) in Sect. 2.2) the expected number of strong swings of an actor are fully counted, while its expected number of weak swings are counted only for a fraction $\epsilon \in[0,1] .{ }^{15}$ Thus, if $\epsilon=1$ weak swings are counted the same as strong swings, while weak swings are not counted at all if $\epsilon=0$.

For every bottom subordinate of actor $i$ the number of $i$ 's strong and weak swings containing that particular bottom subordinate is the same. Therefore, by $\left|S W_{i}^{s}\right|$ we denote the number of strong swings of actor $i$ containing a particular bottom subordinate of $i$, and by $\left|S W_{i}^{w}\right|$ we denote the number of weak swings of actor $i$ containing a particular bottom subordinate of $i$. Since we assumed the proposal to enter every bottom desk with equal probability, the power score $\eta^{\epsilon}(N, D)$ of actor $i$ is given by

$$
\eta_{i}^{\epsilon}(N, D)=\frac{|\bar{S}(i)|}{|K(D)|}\left(\left|S W_{i}^{s}\right|+\epsilon\left|S W_{i}^{w}\right|\right)
$$

where $\bar{S}(i)=(\widehat{S}(i) \cup\{i\}) \cap K(D)$ (and, thus, $\frac{|\bar{S}(i)|}{|K(D)|}$ is the probability that the proposal enters at the desk of one of the bottom subordinates of $i$ or at $i$ 's desk if $i$ is a bottom actor itself).

Next, consider a bottom actor $i \in K(D)$. This actor has one strong and one weak swing. The strong swing results from the action profile where every actor votes yes. Clearly, this action profile yields acceptance, while actor $i$ switching its vote from yes to no yields rejection of the proposal. Bottom actor $i$ also has one weak swing, namely the action profile where it votes no. This leads to a rejection, which might change if the actor votes yes, but not necessarily. Since all bottom actors receive the proposal with equal probability, $i$ 's swings occur with probability $\frac{1}{|K(D)|}$.

Considering the actors $i \notin K(D) \cup\left\{i_{0}\right\}$ who are not at the bottom nor at the top, the same reasoning can be followed for each of their subordinates at the bottom. Hence, for each truncated hierarchy 'middle' actor $i$ is a member of, $i$ has one weak and one strong swing, each occurring with probability $\frac{1}{|K(D)|}$. Finally, the top actor $i_{0}$ has two strong swings for every bottom actor.

Therefore, the power score $\eta^{\epsilon}(N, D)$ of actor $i$ given by (3.1) is equal to

$$
\eta_{i}^{\epsilon}(N, D)= \begin{cases}\frac{2\left|\bar{S}\left(i_{0}\right)\right|}{|K(D)|}=2 & \text { if } i=i_{0} \\ \frac{|\bar{S}(i)|}{|K(D)|}(1+\epsilon) & \text { if } i \neq i_{0}\end{cases}
$$

\footnotetext{
${ }^{14}$ The binary setup in this paper allows us to represent our action-based power score and measure by the digraph of the dominance structure. For a more general representation using extensive game forms we refer to van den Brink and Steffen (2008).

${ }^{15}$ Note that in van den Brink and Steffen (2008) we assume $\epsilon \in(0,1)$ as it appears conceptually reasonable to exclude $\epsilon=0$ and $\epsilon=1$. However, for the axiomatization of the power score and measure it turns out to be useful formally to allow for both extremes. Furthermore, we would like to point out that under our original assumption, i.e., $\epsilon \in(0,1)$, for binary DMMs as discussed in this paper, we have shown that it is not necessary to specify an exact value of $\epsilon$, if one is interested in the power ordering only.
} 
Finally, in order to define a power measure (step (iv) in Sect. 2.2), the power score of every actor is divided by the expected number of action profiles it is a member of. ${ }^{16}$ Every bottom actor (who can receive the proposal from the outside world), and all its superiors are members of a 'positive' profile where they all vote yes, which yields acceptance. Further, in this truncated hierarchy the bottom actor is a member of the 'negative' profiles where it votes no, or it votes yes and one of its superiors votes no (after a sequence of yes votes from intermediate superiors). Each of its superior actors is a member of the 'negative' profiles where every subordinate on the path from this actor to the bottom actor votes yes, and this actor votes no, or this actor votes yes but one of its superiors votes no (after a sequence of yes votes from intermediate superiors), all yielding rejection. (Note that every such action profile consists of a number of consecutive yes-votes, and ends with a no-vote.) Clearly, there are $\left|\widehat{S}^{-1}(i)\right|+1$ of such action profiles for every bottom subordinate of actor $i$. So, for each of its subordinate bottom actors, every actor $i$ is a member of $\left|\widehat{S}^{-1}(i)\right|+2$ action profiles containing this bottom subordinate, and the probability that nature chooses an action profile containing one of $i$ 's bottom actors (or $i$ itself if $i$ is a bottom actor) is $\frac{|\bar{S}(i)|}{|K(D)|}$. This yields the $\hat{\beta}^{\epsilon}$-power measure

$$
\hat{\beta}_{i}^{\epsilon}(N, D)=\frac{\eta_{i}^{\epsilon}(N, D)}{\frac{|\bar{S}(i)|}{|K(D)|}\left(\left|\widehat{S}^{-1}(i)\right|+2\right)}= \begin{cases}\frac{2}{1(0+2)}=1 & \text { if } i=i_{0} \\ \frac{\frac{|S(i)|}{|K(D)|}(1+\epsilon)}{\frac{|S(i)|}{|K(D)|}\left(\left|\widehat{S}^{-1}(i)\right|+2\right)}=\frac{(1+\epsilon)}{\left(\left|\widehat{S}^{-1}(i)\right|+2\right)} & \text { if } i \neq i_{0} .\end{cases}
$$

Note that the composition of the denominator of this power measure shows a significant difference compared to the denominator of the well-known Banzhaf (1965) measure for simultaneous voting situations. To obtain the Banzhaf measure from the Banzhaf score for an actor $i$ its score is divided by $2^{n-1}$ (see Felsenthal and Machover 1998). Representing the voting situation by a simple game being a 'one-sided' model based upon so-called 'winning coalitions' this number corresponds to the number of coalitions actor $i$ is a member of and in which all members vote yes. In terms of action profiles this is equal to the number of all action profiles containing actor $i$ and in which $i$ votes yes. However, in principle, for simultaneous voting $2^{n-1}$ could also be interpreted to be the number of coalitions actor $i$ is a member of and in which all members vote no (losing coalitions) or, in terms of action profiles, as the number of all action profiles containing actor $i$ and in which $i$ votes no. Which interpretation is used makes no difference to the power ascribed by the Banzhaf measure as under a simultaneous voting situation the number of both types of coalitions (and action profiles) is equal. However, this equality no longer holds for sequential voting situations. We showed above that in sequential voting, for each of its subordinate bottom actors an actor $i$ has one action profile in which it votes no (in which, moreover, $i \neq i_{0}$ has a positive weak swing and $i=i_{0}$ has a positive strong swing), and $\left|\widehat{S}^{-1}(i)\right|+1$ action profiles in which $i$ votes yes (in one of those $i$ has a positive strong swing). Thus, following the idea of the Banzhaf measure and modifying the denominator in (3.3) by dividing the power score $\eta_{i}^{\epsilon}(N, D)$ by the number of action profiles in which $i$ votes yes or $i$ votes no yields

\footnotetext{
${ }^{16}$ In the more general setting considered in van den Brink and Steffen (2008) the denominator is the expected number of alternative actions in action profiles actor $i$ is a member of. Since in the binary voting model considered here an actor can choose between two actions only, this denominator boils down to the expected number of action profiles $i$ is a member of. In order to obtain this expected number, the action profiles of each truncated hierarchy $i$ is a member of are weighted by the probability that this truncated hierarchy occurs, i.e., by the probability that a proposal enters at the desk of the bottom actor of this truncated hierarchy.
} 
two different power measures. If we would define the measure by dividing the power score by the expected number of action profiles in which an actor votes no, then the measure is equal to the power score, since for every subordinate bottom actor there is only one such an action profile. However, the $\beta^{\epsilon}$-measure that is obtained by dividing the power score by the expected number of action profiles in which an actor votes yes is not equal to the power score, and is given by

$$
\beta_{i}^{\epsilon}(N, D)=\frac{\eta_{i}^{\epsilon}(N, D)}{\frac{|\bar{S}(i)|}{|K(D)|}\left(\left|\widehat{S}^{-1}(i)\right|+1\right)}= \begin{cases}\frac{2}{1}=2 & \text { if } i=i_{0} \\ \frac{\frac{\bar{S}(i) \mid}{|K(D)|}(1+\epsilon)}{\frac{|\bar{S}(i)|}{|K(D)|}\left(\left|\widehat{S}^{-1}(i)\right|+1\right)}=\frac{(1+\epsilon)}{\left(\left|\widehat{S}^{-1}(i)\right|+1\right)} & \text { if } i \neq i_{0} .\end{cases}
$$

Example 2 For the hierarchy of Example 1 the power score and measures, respectively, are given by $\eta^{\epsilon}(N, D)=(1+\epsilon, 2), \hat{\beta}^{\epsilon}(N, D)=\left(\frac{1}{3}(1+\epsilon), 1\right)$ and $\beta^{\epsilon}(N, D)=\left(\frac{1}{2}(1+\epsilon), 2\right)$.

In the next sections we present axiomatic characterizations of the $\eta^{\epsilon}$-score and $\beta^{\epsilon}$ measure of power under the One Desk Model.

\section{Axiomatizations of the power score}

In this section we provide axiomatizations of the power score $\eta^{\epsilon}$ given by (3.2). We first discuss the extreme cases $\epsilon \in\{0,1\}$, from which the general result follows. In the following axioms $f$ denotes a generic power score or measure.

\subsection{The case $\epsilon=1$}

We begin with the special case where strong and weak swings are assigned equal weight. For $\epsilon=1$ the power score (3.2) equals

$$
\eta_{i}^{1}(N, D)=\frac{2|\bar{S}(i)|}{|K(D)|} \quad \text { for all } i \in N .
$$

We provide two axiomatizations of the power score $\eta^{1}$. The first one uses the following three axioms. As mentioned earlier, our view of power is based on a definition of power as an ability or capacity which exists whether it is exercised or not. What counts is what an actor is able to do in its position in the hierarchy if a proposal reaches the actor. Thus, its power is independent of the choices of his successors (in the dominance structure). Furthermore, according to our definition of a swing the top has two (strong) swings. Hence, we postulate that the top actor $i_{0}$ should have a power score of two having in mind that the score of an actor is the result of the aggregation of its individual power ascription (step (iii) in Sect. 2.2).

Axiom 4.1 (Normalization) For top actor $i_{0}$ it holds that $f_{i_{0}}(N, D)=2$.

Note that due to the fact that we truncate all strict hierarchies which are not already a line into overlapping lines with the top being the unique actor who is a member of all truncated hierarchies, the probabilities determining which bottom actor receives a new proposal under the One Desk Model do not affect the power score of the top.

The principle of truncation of hierarchies is formulated in the following axiom which states that the power score of an actor in a hierarchy is equal to the average of its power 
scores in all truncated hierarchies. Every bottom actor $j \in K(D)$ is associated with a truncated hierarchy $\left(\widehat{S}^{-1}(j) \cup\{j\}, D\left(\widehat{S}^{-1}(j) \cup\{j\}\right)\right)$ which consists of this bottom actor with all its superiors and the dominance relations between them. We denote by $\mathcal{T}_{D}=$ $\left\{\left(\widehat{S}^{-1}(j) \cup\{j\}, D\left(\widehat{S}^{-1}(j) \cup\{j\}\right)\right)\right\}_{j \in K(D)}$ the set of all truncated hierarchies in $(N, D) .{ }^{17}$ For $i \in N$ we denote by $\mathcal{T}_{D}^{i}=\left\{(T, D(T)) \in \mathcal{T}_{D} \mid i \in T\right\}=\left\{\left(\widehat{S}^{-1}(j) \cup\{j\}, D\left(\widehat{S}^{-1}(j) \cup\{j\}\right)\right\}_{j \in \bar{S}(i)}\right.$ the set of all truncated hierarchies that contain actor $i \in N$.

Axiom 4.2 (Truncation) For every $i \in N$ it holds that $f_{i}(N, D)=\frac{1}{|K(D)|} \sum_{(T, D(T)) \in \mathcal{T}_{D}^{i}} f_{i}(T, D(T))$.

The next axiom puts lower- and upper bounds on the power score of an actor and its successors. First, we require some kind of structural monotonicity in the sense that actors 'higher' in the structure have a power score at least equal to that of their 'lower' successors. Second, we require that the power score of an actor cannot be more than the sum of the power scores of all its successors. This yields some kind of power balance in which an actor can have a higher power score than its successors, but the successors are guaranteed to have power by assuming that together they will have at least the same power score as their common predecessor. ${ }^{18}$

Axiom 4.3 (Bound property) For every $i \in N \backslash K(D)$ it holds that

(i) $f_{i}(N, D) \geq f_{j}(N, D)$ for all $j \in S(i)$, and

(ii) $f_{i}(N, D) \leq \sum_{j \in S(i)} f_{j}(N, D)$.

In the case of a line-hierarchy $(N, D)$ (i.e., $|S(i)|=1$ for all $i \in N \backslash K(D)$ ) the bound property determines that all scores are equal, and by normalization all scores are determined to be equal to 2 . Then truncation determines the power scores for arbitrary hierarchies. Since it is straightforward to verify that $\eta^{1}$ satisfies these three axioms, we state the following axiomatization without further proof. ${ }^{19}$

Theorem 4.4 A power score $f$ is equal to $\eta^{1}$, if and only if it satisfies normalization, truncation, and the bound property.

It seems that truncation and the bound property are rather strong axioms to require from a power score. Therefore we suggest the following three alternative axioms. The first one is the axiom of symmetry which is standard.

Axiom 4.5 (Symmetry) If $S(i)=S(j)$ and $S^{-1}(i)=S^{-1}(j)$, then $f_{i}(N, D)=f_{j}(N, D)$.

The second axiom is an independence axiom. This follows Arrow's famous 'independence of irrelevant alternatives' axiom in social choice theory. Here we assume that if we organize the same set of bottom actors, the power of an actor $i$ depends only on the structure of its superiors and subordinates. With this we mean that as long as $i$ and all its subordinates

\footnotetext{
${ }^{17}$ Recall from Sect. 2.3 that $D(T)$ denotes the subgraph of $D$ restricted to $T \subseteq N$.

${ }^{18} \mathrm{~A}$ similar property for wages in firm hierarchies (with constant span of control and identical workers) is assumed by Williamson (1967) and motivated by van den Brink (2008).

${ }^{19}$ The uniqueness part of this theorem also follows from the proof of Theorem 4.9.
} 
have the same successors, and $i$ and all its superiors have the same predecessors, the power score of $i$ does not change.

Axiom 4.6 (Independence of outside organization) Consider two hierarchies $(N, D)$, $\left(N^{\prime}, D^{\prime}\right)$ with $i \in N \cap N^{\prime}$. If (i) $S_{D}(j)=S_{D^{\prime}}(j)$ for all $j \in \widehat{S}_{D}(i) \cup\{i\}$, (ii) $S_{D}^{-1}(j)=S_{D^{\prime}}^{-1}(j)$ for all $j \in \widehat{S}_{D}^{-1}(i) \cup\{i\}$, and (iii) $K(D)=K\left(D^{\prime}\right)$, then $f_{i}(N, D)=f_{i}\left(N^{\prime}, D^{\prime}\right)$.

Finally, we reflect the principle of truncation of the hierarchy (with overlapping memberships) by requiring that the power score of a non-bottom actor equals the sum of the powers of its successors.

Axiom 4.7 (Successor-sum property) For every $i \in N \backslash K(D)$ it holds that $f_{i}(N, D)=$ $\sum_{j \in S(i)} f_{j}(N, D)$.

So, the successor-sum property relates the power score of an actor $i$ to those of its successors in the hierarchy, while truncation (Axiom 4.2) relates $i$ 's score to its own scores in all truncated hierarchies.

Since the changes made in the hierarchy according to the independence of outside organization axiom do not change the structure of the truncated hierarchies that involve actor $i$, it is obvious that truncation implies independence of outside organization. Further, truncation and the bound property together imply the successor-sum property.

Proposition 4.8 If power score $f$ satisfies truncation and the bound property, then it satisfies the successor-sum property.

Proof Suppose that power score $f$ satisfies truncation and the bound property. Consider a hierarchy $(N, D)$ and an actor $h \in N$ with $S(h) \neq \emptyset$.

For every truncated hierarchy $(T, D(T)) \in \mathcal{T}_{D}^{h}$ the bound property implies that all actors in $T$ have the same power score. In particular, actors $h$ and $j \in S(h) \cap T$ have the same power score. Since $\{\bar{S}(j)\}_{j \in S(h)}$ is a partition of $\bar{S}(h)$, the truncation property implies that $f_{h}(N, D)=\sum_{j \in S(h)} f_{j}(N, D)$. So, $f$ satisfies the successor-sum property.

Replacing, in Theorem 4.4, the truncation and bound properties by symmetry, independence of outside organization and the successor-sum property, also yields an axiomatization of the power score $\eta^{1}$.

Theorem 4.9 A power score $f$ is equal to $\eta^{1}$, if and only if it satisfies normalization, symmetry, independence of outside organization, and the successor-sum property.

Proof It is straightforward to verify that $\eta^{1}$ satisfies normalization, symmetry, and independence of outside organization. The successor-sum property follows since by $\{\bar{S}(j)\}_{j \in S(i)}$ being a partition of $\bar{S}(i)$, we have that $\sum_{j \in S(i)} \eta_{j}^{1}(N, D)=\sum_{j \in S(i)} \frac{2|\bar{S}(j)|}{|K(D)|}=\frac{2|\bar{S}(i)|}{|K(D)|}=$ $\eta_{i}^{1}(N, D)$.

To show uniqueness, assume that the power score $f$ satisfies the four axioms, and consider a hierarchy $(N, D)$. Define a reduced hierarchy $\left(N^{0}, S^{0}\right)$ by $N^{0}=\left\{i_{0}\right\} \cup K(D)$, $S^{0}\left(i_{0}\right)=K(D)$ and $S^{0}(j)=\emptyset$ for all $j \in K(D)$; see Figs. 1 and 2 as an illustration of deleting middle actors.

Normalization implies that $f_{i_{0}}\left(N^{0}, S^{0}\right)=2$. Symmetry implies that all actors in $K(D)$ have the same power score in $\left(N^{0}, S^{0}\right)$. Since the successor-sum property implies that the 
Fig. 1 A hierarchy $(N, D)$

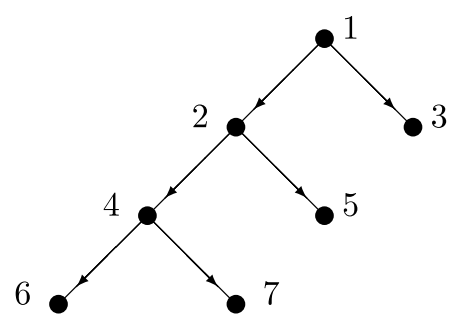

Fig. 2 Hierarchy $\left(N^{0}, S^{0}\right)$

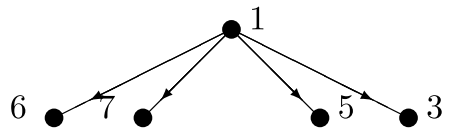

Fig. 3 Hierarchy $\left(N^{6}, S^{6}\right)$

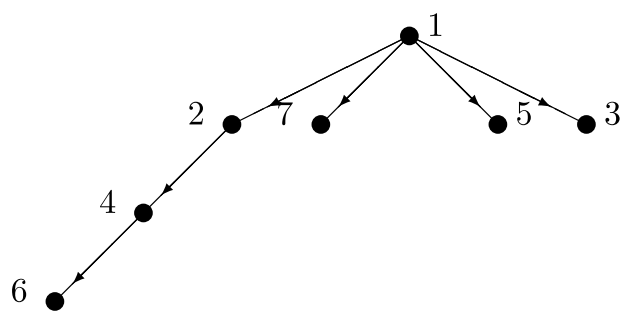

sum of the powers of all actors in $K(D)$ should add up to the power score of the top $i_{0}$, it holds that $f_{j}\left(N^{0}, S^{0}\right)=\frac{2}{|K(D)|}$ for all $j \in K(D)$.

Next take an $i \in K(D)$. Define a hierarchy $\left(N^{i}, S^{i}\right)$ by $N^{i}=K(D) \cup \widehat{S}^{-1}(i)$ (note that this implies that $i_{0} \in N^{i}$ ) and

$$
S^{i}(j)= \begin{cases}(K(D) \backslash\{i\}) \cup\left(S\left(i_{0}\right) \cap\left(\widehat{S}^{-1}(i) \cup\{i\}\right)\right) & \text { if } j=i_{0} \\ S(j) \cap\left(\widehat{S}^{-1}(i) \cup\{i\}\right) & \text { if } j \in \widehat{S}^{-1}(i) \backslash\left\{i_{0}\right\} \\ \varnothing & \text { otherwise; }\end{cases}
$$

see Fig. 3 for an example. Normalization implies that $f_{i_{0}}\left(N^{i}, S^{i}\right)=2$.

Independence of outside organization implies that $f_{j}\left(N^{i}, S^{i}\right)=f_{j}\left(N^{0}, S^{0}\right)=\frac{2}{|K(D)|}$ for all $j \in K(D) \backslash\{i\}$.

Since the successor-sum property implies that the sum of the powers of all actors in $K(D) \backslash\{i\}$ and that of the actor in $S\left(i_{0}\right) \cap\left(\widehat{S}^{-1}(i) \cup\{i\}\right)$ should add up to the given power of the top $i_{0}$, i.e., $f_{i_{0}}\left(N^{i}, D^{i}\right)=2$, it follows that $f_{j}\left(N^{i}, S^{i}\right)=\frac{2}{|K(D)|}$ for $j \in$ $S\left(i_{0}\right) \cap\left(\widehat{S}^{-1}(i) \cup\{i\}\right)$. Repeated application of the successor-sum property further determines that $f_{j}\left(N^{i}, S^{i}\right)=\frac{2}{|K(D)|}$ for all $j \in \widehat{S}^{-1}(i) \backslash\left\{i_{0}\right\}$ and, thus, eventually $f_{i}\left(N^{i}, S^{i}\right)=\frac{2}{|K(D)|}$ is determined.

Independence of outside organization then implies that $f_{i}(N, D)=f_{i}\left(N^{i}, S^{i}\right)=$ $\frac{2}{|K(D)|}=\eta_{i}^{1}(N, D)$.

Hence, all $f_{i}(N, D)$ are then determined for $i \in K(D)$. The successor-sum property, finally, determines the power score for all other actors and, thus, $f(N, D)$ is uniquely determined. 
Logical independence of the axioms in Theorems 4.4 and 4.9 is relegated to the Appendix.

\subsection{The case $\epsilon=0$}

Next, we consider the other extreme case where weak swings are assigned weight zero. Compared to the case $\epsilon=1$ discussed above, all non-top actors $i \neq i_{0}$ 'lose' their weak swings, i.e., the swings where they vote no and their subordinates who receive the proposal on their desk (if they exist) voted yes. But they still have their strong swings, where every actor involved voted yes. Since for the top actor $i_{0}$ all swings are strong, for $\epsilon=0$ the power score given by (3.2) is equal to

$$
\eta_{i}^{0}(N, D)= \begin{cases}\eta_{i}^{1}(N, D) & \text { if } i=i_{0} \\ \frac{|S(i)|}{|K(D)|}=\frac{1}{2} \eta_{i}^{1}(N, D) & \text { for all } i \neq i_{0} .\end{cases}
$$

For $\epsilon=0$ the axioms of normalization, symmetry and independence of outside organization still hold. The successor-sum property holds only outside the top.

Axiom 4.10 (Outside the top successor-sum property) For every $i \in N \backslash\left(K(D) \cup\left\{i_{0}\right\}\right)$ it holds that $f_{i}(N, D)=\sum_{j \in S(i)} f_{j}(N, D)$.

The possibility of the top to finalize the decision to a yes (if it gets the opportunity to vote) is now reflected in a doubling of the sum of the powers of its subordinates.

Axiom 4.11 (Top successor-sum property) For top actor $i_{0}$ it holds that $f_{i_{0}}(N, D)=$ $2 \sum_{j \in S\left(i_{0}\right)} f_{j}(N, D)$.

Replacing the successor-sum property by these two axioms in Theorem 4.9 characterizes $\eta^{0}$.

Theorem 4.12 A power score $f$ is equal to $\eta^{0}$, if and only if it satisfies normalization, symmetry, independence of outside organization, the outside the top successor-sum property, and the top successor-sum property.

The proof is similar to the proof of Theorem 4.9 and is therefore omitted. The only difference is that where the successor-sum property is used concerning the top actor, now the top successor-sum property has to be used.

It is straightforward to verify that the power score $\eta^{0}$ also satisfies truncation. However, the bound property must be adapted, taking the 'special' top position into account.

Axiom 4.13 (Outside the top bound property) For every $i \in N \backslash\left(K(D) \cup\left\{i_{0}\right\}\right)$ it holds that

(i) $f_{i}(N, D) \geq f_{j}(N, D)$ for all $j \in S(i)$, and

(ii) $f_{i}(N, D) \leq \sum_{j \in S(i)} f_{j}(N, D)$.

Axiom 4.14 (Top bound property) For top actor $i_{0}$ it holds that

(i) $f_{i_{0}}(N, D) \geq 2 f_{j}(N, D)$ for all $j \in S\left(i_{0}\right)$, and

(ii) $f_{i_{0}}(N, D) \leq 2 \sum_{j \in S\left(i_{0}\right)} f_{j}(N, D)$. 
We state the following result without proof.

Theorem 4.15 A power score $f$ is equal to $\eta^{0}$, if and only if it satisfies normalization, truncation, the outside the top bound property, and the top bound property.

\subsection{The case $\epsilon \in[0,1]$}

Finally, let us consider the general case. From the two extreme cases considered in the previous two subsections we can derive that for $\epsilon \in[0,1]$ the power score is given by

$$
\eta^{\epsilon}(N, D)=\eta^{0}(N, D)+\epsilon\left(\eta^{1}(N, D)-\eta^{0}(N, D)\right)=\epsilon \eta^{1}(N, D)+(1-\epsilon) \eta^{0}(N, D),
$$

and, thus, is a convex combination of $\eta^{0}$ and $\eta^{1}$. Taking account of $\epsilon \in[0,1]$ with respect to the top successor-sum property in a straightforward way, we obtain the following axiom.

Axiom 4.16 ( $\epsilon$-top successor-sum property) For top actor $i_{0}$ it holds that $f_{i_{0}}(N, D)=$ $\frac{2}{1+\epsilon} \sum_{j \in S\left(i_{0}\right)} f_{j}(N, D)$.

Replacing the top successor-sum property by this axiom in Theorem 4.12 characterizes $\eta^{\epsilon}$.

Theorem 4.17 A power score $f$ is equal to $\eta^{\epsilon}, \epsilon \in[0,1]$, if and only if it satisfies normalization, symmetry, independence of outside organization, the outside the top successor-sum property, and the $\epsilon$-top successor-sum property.

The proof is similar to the proofs of Theorems 4.9 and 4.12, and is therefore omitted.

Recall that $\epsilon \in[0,1]$ is the fraction with which weak swings are counted in obtaining the power score of each actor, given that every strong swing is counted full (i.e., with weight 1 ). Therefore, the choice of $\epsilon$ depends on how important weak swings are in the aggregation of individual power ascriptions. Although an analysis of this is beyond the scope of this paper, the importance of weak swings may depend on the attitude towards risk in a society. For example, in a risk averse society, one only counts what one has for sure and thus, applying this idea to swings, only counts strong swings (i.e., $\epsilon=0$ ). In the other extreme of a risk loving society, all swings are counted equally (i.e., $\epsilon=1$ ). In this context, referring to Axiom 4.16, the attitude towards risk in a society is also reflected in the excess of power of the top actor compared to the sum of powers of its successors: the more risk averse a society is the more powerful is the top actor compared to the other actors.

Also the top bound property can be generalized.

Axiom 4.18 ( $\epsilon$-top bound property) For top actor $i_{0}$ it holds that

(i) $f_{i_{0}}(N, D) \geq \frac{2}{1+\epsilon} f_{j}(N, D)$ for all $j \in S\left(i_{0}\right)$, and

(ii) $f_{i_{0}}(N, D) \leq \frac{2}{1+\epsilon} \sum_{j \in S\left(i_{0}\right)} f_{j}(N, D)$.

We state the following without proof.

Theorem 4.19 A power score $f$ is equal to $\eta^{\epsilon}$, if and only if it satisfies normalization, truncation, the outside the top bound property, and the $\epsilon$-top bound property. 


\section{An axiomatization of the power measure}

In this section we adapt the axioms of the previous section in order to obtain an axiomatization of the power measure $\beta^{\epsilon}$ given by (3.4).

\subsection{The case $\epsilon=1$}

Again, we first consider the case $\epsilon=1$ where all weak swings are fully counted. Substituting in (3.4) the power score $\eta^{1}$ as given by (4.5) yields the power measure

$$
\beta_{i}^{1}(N, D)=\frac{\eta_{i}^{1}(N, D)}{\frac{|\bar{S}(i)|}{|K(D)|}\left(\left|\widehat{S}^{-1}(i)\right|+1\right)}=\frac{2}{\left|\widehat{S}^{-1}(i)\right|+1} \quad \text { for all } i \in N
$$

To characterize this power measure we first consider what properties of the power score $\eta^{1}$ it satisfies. It is easy to verify that it satisfies normalization, symmetry, and independence of outside organization. ${ }^{20}$ It does not satisfy the successor-sum property.

With respect to the independence axiom, $\beta^{1}$ even satisfies a stronger independence axiom where we do not require the set of bottom actors to be the same. Moreover, it does not depend on the successors of subordinates.

Axiom 5.1 (Strong independence of outside organization) Consider two hierarchies $(N, D),\left(N^{\prime}, D^{\prime}\right)$ with $i \in N \cap N^{\prime}$. If $S_{D}^{-1}(j)=S_{D^{\prime}}^{-1}(j)$ for all $j \in \widehat{S}_{D}^{-1}(i)$, then $f_{i}(N, D)=$ $f_{i}\left(N^{\prime}, D^{\prime}\right)$.

Note that deleting the requirement $K(D)=K\left(D^{\prime}\right)$ can be interpreted as the power measure being not dependent on the probabilities with which bottom actors are chosen to receive the proposal (see also Sect. 6).

For the axiomatization we add another axiom which discusses a special type of regular hierarchies. We call a hierarchy a regular hierarchy if the top actor has two successors and at every level (except the bottom level) the number of successors of the actors at that level is one more than that of the direct superior level. So, a hierarchy $(N, D)$ is regular if $|S(i)|=$ $\left|\widehat{S}^{-1}(i)\right|+2$ for all $i \in N \backslash K(D)$.

We define an office as the set of successors of the same predecessor, i.e., every set $S(i)$ of successors of the same actor $i \in N \backslash K(D)$ is an office. Here, we also consider the singleton $\left\{i_{0}\right\}$ as an office and denote the set of all offices in hierarchy $(N, D)$ by $\mathcal{P}(D)=\{S(i) \mid i \in$ $N \backslash K(D)\} \cup\left\{\left\{i_{0}\right\}\right\}$.

Making use of the above definitions the next axiom says that the total power in any office in a regular hierarchy is the same.

Axiom 5.2 (Regularity) If $(N, D)$ is a regular hierarchy, then $\sum_{i \in P} f_{i}(N, D)=$ $\sum_{i \in P^{\prime}} f_{i}(N, D)$ for every pair of offices $P, P^{\prime} \in \mathcal{P}(D)$.

Together with normalization these axioms characterize the power measure $\beta^{1}$.

\footnotetext{
${ }^{20}$ It does satisfy another truncation property where the power measure of an actor $i$ is determined by the average taken over all truncated hierarchies containing $i$ and not all truncated hierarchies as done for the power score, i.e., $f_{i}(N, D)=\frac{1}{|\bar{S}(i)|} \sum_{j \in \bar{S}(i)} f\left(\widehat{S}^{-1}(j) \cup\{j\}, D\left(\widehat{S}^{-1}(j) \cup\{j\}\right)\right)$.
} 
Theorem 5.3 A power measure $f$ is equal to $\beta^{1}$, if and only if it satisfies normalization, strong independence of outside organization, and regularity.

Proof It is straightforward to verify that $\beta^{1}$ satisfies normalization and strong independence of outside organization. Consider a regular hierarchy $(N, D)$ with $i \in P \in \mathcal{P}(D)$. By regularity of the hierarchy, $|P|=\left|\widehat{S}^{-1}(i)\right|+1$, and, thus, $\sum_{i \in P} \beta_{i}^{1}(N, D)=\sum_{i \in P} \frac{2}{\left|\widehat{S}^{-1}(i)\right|+1}=$ $\left(\left|\widehat{S}^{-1}(i)\right|+1\right) \cdot\left(\frac{2}{\left|\widehat{S}^{-1}(i)\right|+1}\right)=2=\beta_{i_{0}}^{1}(N, D)$, showing that $\beta^{1}$ satisfies regularity.

To show uniqueness, assume that the power measure $f$ satisfies the three axioms and consider hierarchy $(N, D)$.

Take an actor $i \in N \backslash\left\{i_{0}\right\}$ and define the reduced hierarchy $\left(N^{i}, D^{i}\right)$ by $N^{i}=\{i\} \cup$ $\widehat{S}^{-1}(i)$ and $D^{i}=D\left(N^{i}\right)$. Assume a set of actors $\bar{N}^{i} \supset N^{i}$ with $\left|\bar{N}^{i}\right|=1+\sum_{k=1}^{\left|\widehat{S}^{-1}(i)\right|} k(k+1)$ and $\bar{D}^{i}$ such that $\left(\bar{N}^{i}, \bar{D}^{i}\right)$ is a regular hierarchy with $S_{D^{i}}(j) \subseteq S_{\bar{D}^{i}}(j)$ for all $j \in \widehat{S}_{D^{i}}^{-1}(i)$. (Note that we choose $\left|\bar{N}^{i}\right|$ such that a regular hierarchy exists.) Normalization implies that $f_{i_{0}}\left(\bar{N}^{i}, \bar{D}^{i}\right)=2$. Let $S^{-1}(i)=\{h\}$. Then regularity implies that

$$
\sum_{j \in S_{\bar{D}^{i}}(h)} f_{j}\left(\bar{N}^{i}, \bar{D}^{i}\right)=2 .
$$

Next, we show that $f_{i}\left(\bar{N}^{i}, \bar{D}^{i}\right)=\frac{2}{\left|\widehat{S}^{-1}(i)\right|+1}$. Take an actor $j \in S_{\bar{D}^{i}}(h) \backslash\{i\}$. Now consider an actor $z \in \mathbb{N} \backslash \bar{N}^{i}$ and let $\left(N^{\prime}, D^{\prime}\right)$ be given by $N^{\prime}=\left(\bar{N}^{i} \backslash\{j\}\right) \cup\{z\}$ and

$$
S_{D^{\prime}}(g)= \begin{cases}\left(S_{\bar{D}^{i}}(h) \backslash\{j\}\right) \cup\{z\} & \text { if } g=h \\ S_{\bar{D}^{i}}(g) & \text { if } g \in \bar{N}^{i} \backslash\{h, j\} \\ S_{\bar{D}^{i}}(j) & \text { if } g=z .\end{cases}
$$

Strong independence of outside organization implies that

$$
f_{g}\left(N^{\prime}, D^{\prime}\right)=f_{g}\left(\bar{N}^{i}, \bar{D}^{i}\right) \quad \text { for all } g \in S_{\bar{D}^{i}}(h) \backslash\{j\} .
$$

By regularity it then follows that

$$
f_{z}\left(N^{\prime}, D^{\prime}\right)=f_{j}\left(\bar{N}^{i}, \bar{D}^{i}\right)
$$

Now define $(\tilde{N}, \widetilde{D})$ by $\widetilde{N}=\left(N^{\prime} \backslash\{i\}\right) \cup\{j\}$ and

$$
S_{\widetilde{D}}(g)= \begin{cases}\left(S_{D^{\prime}}(h) \backslash\{i\}\right) \cup\{j\} & \text { if } g=h \\ S_{D^{\prime}}(g) & \text { if } g \in N^{\prime} \backslash\{h, i\} \\ S_{D^{\prime}}(i) & \text { if } g=j .\end{cases}
$$

As before, strong independence of outside organization implies that

$$
f_{g}(\tilde{N}, \tilde{D})=f_{g}\left(N^{\prime}, D^{\prime}\right) \quad \text { for all } g \in S_{D^{\prime}}(h) \backslash\{i\} .
$$

By regularity it then follows that

$$
f_{j}(\tilde{N}, \widetilde{D})=f_{i}\left(N^{\prime}, D^{\prime}\right)
$$


Making use of (5.8) we obtain

$$
f_{j}\left(\bar{N}^{i}, \bar{D}^{i}\right)=f_{j}(\tilde{N}, \widetilde{D})=f_{i}\left(N^{\prime}, D^{\prime}\right)=f_{i}\left(\bar{N}^{i}, \bar{D}^{i}\right),
$$

where the first equality follows from strong independence of outside organization, the second from (5.8) and the third from (5.7). This holds for all $j \in S_{\bar{D}^{i}}(h) \backslash\{i\}$.

Since by regularity of $\left(\bar{N}^{i}, \bar{D}^{i}\right)$ we have $\left|S_{\bar{D}^{i}}(h)\right|=\left|\widehat{S}^{-1}(i)\right|+1$, it follows that $f_{i}\left(\bar{N}^{i}, \bar{D}^{i}\right)=\frac{2}{\left|\widehat{S}^{-1}(i)\right|+1}$. Independence of outside organization finally implies that $f_{i}(N, D)=f_{i}\left(\bar{N}^{i}, \bar{D}^{i}\right)=\frac{2}{\left|\widehat{S}^{-1}(i)\right|+1}$.

Logical independence is again relegated to the Appendix.

\subsection{The case $\epsilon=0$}

Considering the case that weak swings are assigned weight zero, we obtain the power measure $\beta^{0}$ given by

$$
\begin{aligned}
\beta_{i}^{0}(N, D) & =\frac{\eta_{i}^{0}(N, D)}{\frac{|\bar{S}(i)|}{|K(D)|}\left(\left|\widehat{S}^{-1}(i)\right|+1\right)} \\
& = \begin{cases}2 & \text { if } i=i_{0} \\
\frac{1}{\left|\widehat{S}^{-1}(i)\right|+1} & \text { for all } i \neq i_{0} .\end{cases}
\end{aligned}
$$

This power measure satisfies the axioms of Theorem 5.3 except regularity. It still satisfies regularity as long as we do not consider the singleton $\left\{i_{0}\right\}$ as an office. Hence, for the top actor we need a separate regularity condition. We denote by $\overline{\mathcal{P}}(D)=\mathcal{P}(D) \backslash\left\{\left\{i_{0}\right\}\right\}$ the set of all offices except the top-office.

Axiom 5.4 (Top-regularity) If $(N, D)$ is a regular hierarchy, then $f_{i_{0}}(N, D)=$ $2 \sum_{i \in P} f_{i}(N, D)$ for every office $P \in \overline{\mathcal{P}}(D)$.

Replacing regularity in Theorem 5.3 by top regularity characterizes $\beta^{0}$.

Theorem 5.5 A power measure $f$ is equal to $\beta^{0}$, if and only if it satisfies normalization, strong independence of outside organization, and top-regularity.

Proof It is straightforward to verify that $\beta^{0}$ satisfies these axioms. With respect to uniqueness, normalization fixes the power measure for $i_{0}$. The power measures for the other actors then are determined by top-regularity and strong independence of outside organization similar to the uniqueness proof of Theorem 5.3.

\subsection{The case $\epsilon \in[0,1]$}

Finally, let us consider, again, the general case. From the two extreme cases considered in the previous two subsections we can derive that for $\epsilon \in[0,1]$ the power measure is given by

$$
\beta^{\epsilon}(N, D)=\epsilon \beta^{1}(N, D)+(1-\epsilon) \beta^{0}(N, D) .
$$

For $\epsilon \in[0,1]$ we generalize top-regularity in a similar way as we generalized the top successor-sum property for power scores. 
Axiom 5.6 ( $\epsilon$-Top regularity) If $(N, D)$ is a regular hierarchy, then $f_{i_{0}}(N, D)$ $=\frac{2}{1+\epsilon} \sum_{i \in P} f_{i}(N, D)$ for every office $P \in \overline{\mathcal{P}}(D)$.

Replacing top-regularity in Theorem 5.5 by this axiom characterizes $\beta^{\epsilon}$.

Theorem 5.7 A power measure $f$ is equal to $\beta^{\epsilon}$, if and only if it satisfies normalization, strong independence of outside organization, and $\epsilon$-top regularity.

The proof is similar to the proof of the previous theorems and is therefore omitted.

\section{Generalizations}

\subsection{Generalization of the power score}

So far we assumed that we have no information about the probability with which a proposal enters each bottom desk. This allowed us to apply the principle of insufficient reason assuming that a proposal enters each bottom desk with equal probability (see Sect. 3.1). Now we relax this assumption. We allow for the existence of information regarding the probability with which a proposal enters each bottom desk by permitting any probability distribution $p$ on $K(D)$, i.e., $p_{j} \geq 0$ for all $j \in K(D)$ and $\sum_{j \in K(D)} p_{j}=1$, such that the proposal enters bottom desk $j \in K(D)$ with probability $p_{j}$. Since for every truncated hierarchy with one bottom actor the power score of an actor equals $\left(\left|S W_{i}^{s}\right|+\epsilon\left|S W_{i}^{w}\right|\right)$, we obtain the generalized power score by using the probabilities $p_{j}, j \in K(D)$ and then taking the expected value for each actor:

$$
\eta_{i}^{p, \epsilon}(N, D)=\sum_{j \in \bar{S}(i)} p_{j}\left(\left|S W_{i}^{s}\right|+\epsilon\left|S W_{i}^{w}\right|\right) .
$$

Note that (6.9) reduces to the standard case $\eta_{i}^{\epsilon}$ discussed in earlier sections (see (3.1)), if we make use of the uniform distribution $p_{i}=\frac{1}{|K(D)|}$ for all $i \in K(D)$.

Based upon (6.9) we can now replace (3.2) by

$$
\eta_{i}^{p, \epsilon}(N, D)= \begin{cases}2 \sum_{j \in \bar{S}\left(i_{0}\right)} p_{j}=2 \sum_{j \in K(D)} p_{j}=2 & \text { if } i=i_{0} \\ (1+\epsilon) \sum_{j \in \bar{S}(i)} p_{j} & \text { if } i \neq i_{0} .\end{cases}
$$

Obviously, there is no difference for the top actor since its power score equals 2 for every truncated hierarchy with one bottom actor, so the probability distribution with which the proposal enters the bottom desks is not relevant for the power score of the top actor. The power scores of the other actors do depend on the probabilities with which the proposal enters the bottom desks.

\subsection{Axiomatizations of the generalized power score}

Concerning the axiomatization of the generalized power score we provide here only the case $\epsilon=1$ since the general case follows in a similar way. For $\epsilon=1$ the generalized power score becomes

$$
\eta_{i}^{p, 1}(N, D)=2 \sum_{j \in \bar{S}(i)} p_{j} \quad \text { for all } i \in N
$$


From the axioms of Theorem 4.4 the power score $\eta_{i}^{p, 1}$ satisfies normalization and the bound property. Clearly, it does not satisfy truncation, but this property can be replaced by some kind of expected truncation property stating that (instead of just the average) the power score of an actor in a hierarchy is equal to the expected value of its power scores in all truncated hierarchies. ${ }^{21}$

Axiom 6.1 (Expected truncation) Given probability distribution $p$ over $K(D)$, for every $i \in N$ it holds that $f_{i}(N, D)=\sum_{(T, D(T)) \in \mathcal{T}_{D}^{i}} p_{b(T)} f_{i}(T, D(T))$, where $b(T)$ is the bottom actor in the truncated hierarchy $T$, i.e., $b(T) \in T$ and $S_{D}(b(T))=S_{D(T)}(b(T))=\emptyset$.

Theorem 6.2 Given probability distribution p over $K(D)$, a power score $f$ is equal to $\eta^{p, 1}$, if and only if it satisfies normalization, expected truncation, and the bound property.

Concerning the axioms in Theorem 4.9 it is interesting that the generalized power score also satisfies independence of outside organization and the successor-sum property. In fact, we have a proposition similar to Proposition 4.8. The proof is similar to that of Proposition 4.8 and is therefore omitted.

Proposition 6.3 If power score $f$ satisfies expected truncation and the bound property, then it satisfies the successor-sum property.

Obviously, the generalized power score does not satisfy symmetry. This axiom should change taking account of the non-uniform probabilities. Since we use symmetry only for the star type digraphs as $\left(N_{0}, D_{0}\right)$ in Fig. 2, we can adjust it just for these cases. If two bottom actors have the same predecessors, then their power score is determined by the probabilities with which the proposal enters their desk. ${ }^{22}$

Axiom 6.4 (Symmetric bottom actors) Given probability distribution $p$ over $K(D)$, if $S(i)=S(j)=\emptyset$ and $S^{-1}(i)=S^{-1}(j)$, then $p_{j} f_{i}(N, D)=p_{i} f_{j}(N, D)$.

As for the uniform distribution $p_{i}=\frac{1}{|K(D)|}$ for all $i \in K(D)$, this axiom is weaker than symmetry but is sufficient for the axiomatization in Theorem 4.9. The proof of the following theorem is similar to that of Theorem 4.9 (just applying symmetric bottom actors instead of symmetry) and is therefore omitted. ${ }^{23}$

Theorem 6.5 A power score $f$ is equal to $\eta^{p, 1}$, if and only if it satisfies normalization, symmetric bottom actors, independence of outside organization, and the successor-sum property.

Similar axiomatizations can be given for the case $\epsilon=0$ and the general case $\epsilon \in[0,1]$ by replacing the corresponding axioms in the same way as it was done in Sect. 4 . Hence, all the power scores $\eta^{p, \varepsilon}$ satisfy the generalized expected truncation and symmetric bottom actors properties.

\footnotetext{
${ }^{21}$ Note that expected truncation refers to 'expected truncation with respect to probability distribution $p$ '. However, for convenience we simply refer to this as 'expected truncation' but one should realize that it refers to a given probability distribution over the bottom actors.

${ }^{22}$ Similar as with expected truncation, this axiom depends on the given probability distribution $p$, but, for convenience, we do not refer to $p$ in the name.

${ }^{23}$ The proof can be obtained from the authors on request.
} 


\subsection{Generalization of the power measure}

Finally, let us define a generalized power measure by dividing the generalized power score of an actor by the expected number of action profiles it is a member of and votes yes. Doing this we obtain the power measure

$$
\beta_{i}^{p, \epsilon}(N, D)=\frac{\eta_{i}^{p, \epsilon}(N, D)}{\left(\left|\widehat{S}^{-1}(i)\right|+1\right) \sum_{j \in \bar{S}(i)} p_{j}}= \begin{cases}\frac{2}{1}=2 & \text { if } i=i_{0} \\ \frac{(1+\epsilon) \sum_{j \in \bar{S}(i)} p_{j}}{\left(\left|\widehat{S}^{-1}(i)\right|+1\right) \sum_{j \in \bar{S}(i)} p_{j}}=\frac{(1+\epsilon)}{\left(\widehat{S}^{-1}(i) \mid+1\right)} & \text { if } i \neq i_{0} .\end{cases}
$$

Note that this power measure is the same as the one defined in (3.4). Hence, the values of the power measure do not depend on the probabilities with which the proposal enters the bottom actors. The same holds for the power measure $\hat{\beta}^{\epsilon}$, i.e., if we divide the power score of an actor by the expected number of action profiles this actor is a member of; see (3.3). Since this is what should be expected from a power measure, this further supports our approach of measuring power in sequential DMMs.

\section{Applications}

Among all kinds of organizational architectures, hierarchies can be found in many areas of a society. Typical questions addressed in the literature are whether there exists a tendency of organizational architectures to become hierarchical or how the efficiency of an organization is related to its architecture. ${ }^{24}$ When it comes to the analysis of power in hierarchies we already stated in Sect. 1 that most studies focus on the analysis of one component of positional power only: the authority structure which is based on the bare positions of the actors. Then the analysis is restricted to the study of the abilities to enforce obedience and ignores the DMM in use within a hierarchy, being the second component of the notion of positional power (see Sect. 1). Obviously, ignoring this second component may lead to misleading results regarding the distribution of positional power within organizations (see Sects. 3.2 and 8). In this context we would like to point out that the distribution of positional power has a strong impact on the attitudes and the behavior of the members of an organization, and, thus, on its performance (Daudi 1986; Johnston and Gill 1993; Martin 1998). Moreover, it is crucial when it comes to the ascription of responsibility (Braham and Holler 2008) which is seen as a vital parameter determining the success of an organization (Tannenbaum and Kahn 1957; Chandler 1966).

In the following we will illustrate potential applications of the positional power score and measure defined in this paper. Applications can, in particular, be found in public administration and business, as authority relations are quite common in these areas. ${ }^{25}$ Below, we give an example of a collective-decision making situation for both areas. In both cases actors in a hierarchy have to make a binary decision on an exogenous proposal. The first one is related to business and comes from retail banking. The second one concerns public

\footnotetext{
${ }^{24}$ See, for instance, Mackenzie (1976: 98 ff), Radner (1992: $\left.1391 \mathrm{ff}\right)$, Bolton and Dewatripont (1994), Chwe (2000), and for an early general contribution Simon (1962).

${ }^{25}$ As democratic political decision-making bodies are typically inherently non-hierarchical the applicability of the approach discussed in this paper to such bodies is limited. However, a slightly modified version of our approach could be used in order to analyze the power structures in constitutions containing a mixture of democratic and theocratic elements, such as the current constitution of Iran.
} 
administration and refers to a decision-making situation at a university. In both cases we have chosen a simplified example, although our approach can be applied to a more complex one, for instance, one with more actors being involved.

Following Matthews and Thompson (2008) retail banking can essentially be characterized as granting loans to and receiving deposits from individuals and small businesses. These transactions are typically of small value per transaction but large in volume. Hence, in retail banking many products are highly standardized, i.e., there is only very little room for negotiation between customers and their bank officers. Thus, if a customer approaches its bank officer in a branch with a request, then the bank officer's decision is essentially a binary one and the request of the customer can be regarded to be of an exogenous nature from the bank's point of view. As an example imagine that a customer requests a consumer credit over a certain amount, e.g., for a new car. Such a loan is usually granted on the basis of the customer's creditworthiness, while the characteristics of the investment are usually irrelevant for the decision. When being faced with such a request, the bank officer is usually in a position to reject such a loan. For instance, this may be the case because the customer has recently failed to repay an earlier loan. However, the bank officer is usually not in the position to grant the loan on his own. For this the consent of at least one other staff member is required ('four-eyes-principle'). This is typically a back-office staff member who bears the responsibility for consumer loans within the branch. In addition, if the loan exceeds a certain amount the consent of the head of the branch is also required. Thus, what we have in both cases is a sequential collective decision-making situation in a hierarchy. A corresponding hierarchy for a branch with three bank officers is illustrated by Fig. 4. Note that under the One Desk model which we assume in this paper, a request for consumer credit by one customer would be submitted to one of the bank officers only.

Another context in which our score and measure could be applied is that of student admission at a university level. ${ }^{26}$ When students send their applications for a certain degree

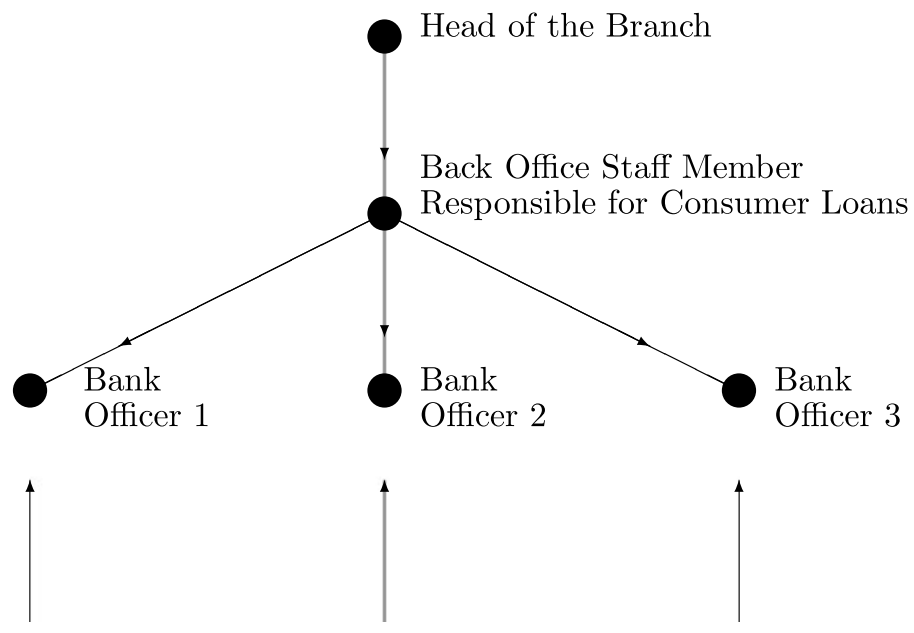

Customer's Requests for Consumer Credits

Fig. 4 A hierarchy in a local branch of a bank

\footnotetext{
${ }^{26}$ See Laruelle and Valenciano (2008) for a similar example.
} 
programme at a university a typical DMM is as follows. When the applications arrive an administrative support staff person will undertake a first screening of the applications and will immediately reject those which appear not to fulfil a set of basic criteria which are often set by the director of the degree programme. For international applicants a typical criterion would be the proficiency in the teaching language. Only after this first selection of candidates has taken place, the director of the degree programme will make a final decision on the remaining set of applicants as to whether they will be admitted or not. Hence, the director of the degree programme has some power over the support staff as the criteria set by her have an influence on the behavior of the support staff by defining boundaries for their decisions. However, within these boundaries the support staff has to decide whether an application will be straightforwardly rejected or whether the application will be forwarded to the director of the degree programme. Again we have a binary decision on an exogenous proposal as the applicants simply submit their applications and based on them it is decided whether an applicant is admitted or not.

\section{The membership-based approaches: a critical appraisal}

In Sect. 3.2 we briefly discussed the general problems of the membership-based approaches when it comes to the analysis of positional power under sequential DMMs which are typical for hierarchical organizations. ${ }^{27}$ In this section we now present a closer examination of these problems by referring separately to each of those membership-based approaches which have been suggested to be applicable for the measurement of positional power in hierarchies. Doing so we also provide a flavor of their construction. ${ }^{28}$

Although the analysis of power in non-hierarchical decision-making bodies has become increasingly popular in the second half of the last century, and the use of tools of game theory for analyzing hierarchical bodies was foreseen many years ago by Morgenstern (1951), Shapley (1962: 66), and Shubik (1962), the analysis of (positional) power in hierarchies has been more or less left by the wayside. ${ }^{29}$

Notable exceptions are the contributions by van den Brink (1994, 1997, 1999, 2001), Gilles et al. (1992), Gilles and Owen (1994), and van den Brink and Gilles (1996) suggesting a Shapley and a Banzhaf permission value. Both can be interpreted as measures of positional power if applied to simple games (see Gilles et al. 1992). ${ }^{30}$ The aspects of positional power in hierarchies are taken into account by representing the decision-making situation as a combination of (i) a simple game representing a binary simultaneous DMM

\footnotetext{
${ }^{27}$ For a more detailed discussion and an example see van den Brink and Steffen (2008).

${ }^{28}$ Note that a comparison of these approaches with ours based on their axiomatization, if existent, would be inappropriate because they are based on simultaneous DMMs, while our approach assumes a sequential DMM.

${ }^{29}$ Note that there exists, in some way, a related field of research in sociology (see, for instance, Tannenbaum and Kahn 1957; Tannenbaum 1961; and Smith and Tannenbaum 1963) concerned with the exercise of control of actors over the collective decisions in hierarchical organizations, but not with bare ability to control. As control is here defined as the effect an actor has in determining the actions of an organization it appears to be either a sub-category of what we call positional power (see Tannenbaum and Kahn 1957) or more or less the same (see Tannenbaum 1961). However, given that these scholars are concerned with the exercise of it they make use of a quite different methodology, i.e., a set of questions to be answered by the members of a hierarchy.

${ }^{30}$ Note that the central aim of their cooperative game theoretic analysis is the creation of allocation mechanisms for the distribution of value within hierarchies, e.g., for payment schemes for employees of a firm.
} 
for a non-hierarchical (or 'flat') decision-making body (let us call this the 'flat DMM'), (ii) a permission structure which takes into account the authority structure represented by the dominance relations of a digraph, and (iii) a decision-making procedure providing the course of actions. All three components are then linked via a permission rule which takes the action profiles of the flat DMM and extends these action profiles which are able to approve a proposal under the flat DMM by taking account of the permission structure and the decision-making procedure, i.e., it adds the actions of the relevant superiors of the actors whose actions are currently included in these profiles. In simple game theoretic parlance this means that each winning coalition of the flat DMM is extended by the relevant superiors of the members of this coalition. This approach implies that principles P1 and P2 discussed in Sect. 2.1 are ignored.

Example 3 Consider a decision-making situation in a hierarchy $(N, D)$ with $N=\{1,2,3\}$ and $D=\{(1,2),(1,3)\}$ and a flat DMM stating that the actors 2 and 3 can, on their own, approve a proposal. Due to the standard monotonicity assumption in a simple game theoretic framework this implies that, also, all supersets of $\{2\}$ and $\{3\}$ can approve the proposal, i.e., $\{1,2\},\{1,3\},\{2,3\}$, and $N$. Now assume a decision-making procedure as defined in Sect. 2.1, i.e., we have a One Desk Model where proposals arrive at bottom desks only. Applying the permission rule taking into account $D$ and the decision-making procedure implies that $\{2\},\{3\}$, and $\{2,3\}$ are no longer in the position to approve a proposal. However, by P1 $N$ should have been excluded as well. Moreover, making use of a simple game theoretic framework which is inherently simultaneous and restricting the view to what different sets of actors can approve ignores also P2 which (as implemented in Sect. 2.1) implies a sequential DMM and an asymmetry of the actors abilities with respect to the rejection of the proposal.

More recently, Shapley and Palamara (2000a, 2000b) have also developed a simple game theoretic framework for measuring positional power in hierarchies which they equate with the notion of responsibility. For the derivation of their power measure they introduce four types of games. They begin with the introduction of a command game. This game is defined for an actor $i$ and informs about which actors dominate $i$ directly. Hence, it corresponds to the set of predecessors $S_{D}^{-1}(i)$ in our setup. The set of all command games within a hierarchy then describes the local authority structure. In order to transfer this information into a global one, Shapley and Palamara define a control game for each actor which identifies the subsets of actors (or coalitions) by which an actor $i$ is controlled. This corresponds to what we call the set of actor $i$ 's superiors $\widehat{S}_{D}^{-1}(i)$. Next, they introduce a task game where they restrict themselves to exogenous tasks with a binary nature. Ignoring the dominance structure of the hierarchy and taking into account P1 it defines the subsets of actors which have the ability to fulfil a certain task, i.e., to effect a certain collective outcome. Finally, they compose the two aforementioned games and call the compound a task control game which is defined for a certain task. For this task it defines which coalitions in a hierarchy can effect an outcome by their collective decision. Making use of the task control game, which itself is also a simple game, Shapley and Palamara suggest that positional power (and responsibility) in a hierarchy can be measured by calculating the Shapley and Shubik (1954) index for this game. However, they do not insist that this is necessarily the most appropriate measure as they mention the Banzhaf (1965) measure as a serious alternative candidate which they do not examine. Again, for the same reason as mentioned above, this approach, also, does not take into account $\mathrm{P} 2$. 
A third approach can be found in Steffen (2002) who suggests the use of Straffin's (1977, 1978) partial homogeneity approach for measuring positional power in hierarchies. This approach which is also based on simple games allows one to partition a set of actors in subgroups of homogeneous actors. It (formally) contains the Banzhaf measure and the ShapelyShubik index as extreme cases. ${ }^{31}$ By its nature this approach allows one to take into account the fact that actors who participate in a decision-making in general have a damatis personae: they are the bearers of predetermined attributes and modes of behavior. That is, actors in hierarchies often play predetermined roles, such as salesman, financial officer, head of external affairs, etc., which are equipped with a bundle of incentive structures. Therefore, it is argued that determining the power of an actor in such a structure must take such information into account, for instance, by partitioning the set of actors into a priori homogeneous subsets, because it is likely that actors with the same incentive structure will act in the same way. ${ }^{32}$ In order to obey the dominance structure Steffen applies an idea similar to Shapley and Palamara and represents the decision-making process as a compound game. Moreover, he shows that this approach, if all actors have the same incentive structure being either all elements of the unique element of the partition that consists of all actors, or being all singletons in the partition that consists of all singletons, is equivalent to van den Brink, Gilles and Owen's measures, i.e., the approach coincides with the Shapley and the Banzhaf permission value, respectively, being applied to simple games. Hence, it suffers from the same pathologies.

Finally, we refer to Berg and Paroush (1998), whose main concern is not the analysis of positional power, but is the analysis of the properties of judgement aggregation in hierarchies. In contrast to the three former approaches, their study to a certain degree ignores the existence of the dominance structure as only the ranking of the actors is taken into account. For each rank they define a majority game and compose those into a product game, i.e., a game where each component has to be won in order to win the whole game. Using this compound game they analyze the probability of a correct collective decision of the hierarchy assuming the existence of an exogenous truth. In the course of the derivation of this probability, they obtain a binomial probability expression which they call a ' $\beta$-number' and which coincides with the Banzhaf measure for their compound game. Having stated this without a proof they point out that, thus, their approach can also be applied to analyze positional power in hierarchies. As Berg and Paroush define a majority game for each rank, their approach does not take into account P1. Moreover, being based on a simple game theoretic framework like the three former approaches, it also ignores P2.

\section{Concluding remarks}

In this paper we applied the power score and measure introduced in van den Brink and Steffen (2008) for general sequential DMMs in hierarchies to the special class of binary voting. As our approach is also applicable to sequential DMMs in general it can also be utilized to analyze other organizational architectures using such DMMs. For example, in many democracies one needs approval of two 'houses' in the parliament, i.e., a 'lower house' and an 'upper house', in order to pass a bill. So, even when both houses vote simultaneously the

\footnotetext{
${ }^{31}$ One arrives at the Banzhaf measure, if one assumes that the partition consists of singletons only, each corresponding to one actor, and at the Shapely-Shubik index, if one assumes that there is one element in the partition only, which then must be the coalition of all actors.

${ }^{32}$ Steffen (2002) uses the term 'act in the same way' in order to point out that he is not talking about 'cooperation' of players in the sense of Aumann and Drèze (1974).
} 
complete DMM is sequential where usually first the 'lower house' votes and only after acceptance the 'upper house' gets to vote. So, when the 'lower house' votes against a proposal then the members in the upper house do not even get the possibility to vote. The sequential nature of voting is even more apparent when, already, the 'lower house' votes sequentially in which case usually the voting stops as soon as the outcome is known. ${ }^{33}$ Considering the case where the 'lower house' and the 'upper house' each vote simultaneously but the 'lower house' votes before the 'upper house', the corresponding hierarchy has all 'lower house' members as bottom actors since they are the ones where proposals enter. Hence, as usual in many policy making government bodies the DMM in place is an All Desk DMM as the proposal enters at all bottom actors simultaneously (see Footnote 13). ${ }^{34}$

Also, in the European Union many issues are decided by different institutions voting sequentially on proposals. The inter-institutional distribution of power between the European Parliament and the Council of Ministers, for example, is studied by Napel and Widgrén (2006). We argued (also in van den Brink and Steffen 2008) that the standard power measures may misrepresent the distribution of power if decision-making is sequential. Although one can analyze power in one government body using a simultaneous voting procedure (see, e.g., Leech 2002 and Felsenthal and Machover 2004 who analyze power in the Council of Ministers) one should realize that for many issues the voting outcome in such a government body is just one step in a larger sequential voting procedure in which, for example, also the European Parliament has to vote. Since power measures are applied in developing voting procedures (for example in various reforms of the EU voting procedures as in the treaties of Nice and Lisbon), a transparent method of power measurement needs to take this into account. Our approach can also be applied to the special type of sequential voting as studied by Sutter (2000), who applies the Banzhaf value to measure voting power in the EU under flexible integration when some countries continue integration among themselves with no commitment for the other EU-members.

We want to conclude this paper with some remarks on the indicated relation of our model to Sah and Stiglitz's $(1985,1986)$ work on hierarchies (see Sect. 3). ${ }^{35}$ To illustrate the relation we make use of some insights by Kaniovski (2008). These allow us to express Sah and Stiglitz's work in a terminology which makes a comparison with our work feasible. ${ }^{36}$ In this sense, we can say that Sah and Stiglitz's work focuses on the relation between the architecture of an organization and its members' collective competence to detect the truth of a proposition, i.e., they have to decide whether a proposition is true or false. In general, a competence is defined as the state of being adequately qualified to do something, which implies that there exists a special type of ability containing an evaluative component in addition

\footnotetext{
${ }^{33}$ For example, in the Netherlands the default is to vote simultaneously, but each member of parliament can demand that voting is sequential in which case the names of the 'lower house' or 'upper house' members are mentioned one by one (in random order) after which they publicly cast their votes.

${ }^{34}$ Note that in other cases a proposal does not enter at all bottom actors, but only at a few or even only at one bottom actor, i.e., we have a Multi or even a One Desk DMM (see Footnote 13). For example, when applying for subsidies the applications usually enter at the bottom of the decision making organization-be it a private or public one-, and eventually after several rounds of selection, the highest level makes a decision on the finally selected applications. For examples from economics see Sah and Stiglitz (1986).

${ }^{35}$ While Sah and Stiglitz's (1986) analysis is restricted to polyarchies and hierarchies with a line structure only, in Sah and Stiglitz (1985) also more complex architectures as investigated in the underlying paper are taken into account.

${ }^{36}$ Kaniovski (2008) in his paper links the theory of voting power to the theory of judgement aggregation. He does not refer to the work of Sah and Stiglitz $(1985,1986)$ which is due to the fact that he was not aware of it.
} 
to the bare ability to make a decision, i.e., to choose a non-empty proper subset of elements out of an outcome set (see van den Brink and Steffen 2008). ${ }^{37}$ In Sah and Stiglitz's case this evaluative component is the ability to detect the truth in addition to the bare ability to choose between elements of the action set containing the elements 'true' and 'false'. Hence, like in our case the action and outcome set is binary. Sah and Stiglitz commence with the assumption that each member of an organization possesses an exogenously given individual competence (ability) to detect the truth. ${ }^{38}$ Via the architecture of the organization, i.e., the dominance structure and the DMM in use, these individual competences (abilities) are then aggregated into a collective competence (ability) of the organization. However, by inserting the members of an organization into their positions, i.e., by the aggregation mechanism via the organizational architecture, their individual bare ability to make a decision is affected by the ability of their positions to make a decision. The latter ability is the subject matter of the underlying paper: positional power. Both abilities together establish the collective competence of the organization. ${ }^{39}$ Thus, in their analysis Sah and Stiglitz implicitly integrate individual and positional abilities without either distinguishing between the components or discussing whether their composition is appropriate or not. However, in doing so they analyze the effect of different organizational architectures on the collective competence of an organization, i.e., they investigate how different distributions of positional power affect the aggregated extent of the exogenously given individual competences of these actors.

To conclude we quote Packel and Deegan (1980), who end their seminal article by saying that 'We believe that there will always be room for simple, well-motivated models of individual and group behavior. If such models are accompanied by coherent schemes of characterizing axioms, they may simultaneously contribute meaningfully to the theory and carve out domains of practical applicability. Von Neumann and Morgenstern never said it would be easy'. We fully agree with this statement and our attempt to solve the problems of the traditional power measures when it comes to the application to sequential DMMs by introducing and axiomatizing a new score and measure should be seen in this light.

Acknowledgements We would like to thank Matthew Braham, Manfred Holler, Martin Leroch, and Gisle Natvik for comments and discussions on forerunners of this paper. Moreover, we are grateful for the detailed and very helpful comments we received from two anonymous referees and the Editor in Chief on two earlier versions of this paper.

Open Access This article is distributed under the terms of the Creative Commons Attribution Noncommercial License which permits any noncommercial use, distribution, and reproduction in any medium, provided the original author(s) and source are credited.

\section{Appendix: Logical independence of the axioms}

In this section we discuss the logical independence of the axioms in Theorems 4.4, 4.9, and 5.3 by providing for each theorem a set of alternative power scores or measures, respectively,

\footnotetext{
${ }^{37}$ Kaniovski (2008) calls this special type of ability 'problem solving power'. Unfortunately, he fails to provide a rigorous analysis whether this terminology withstands a deeper conceptual examination. Therefore, we refrain from making use of it here.

${ }^{38}$ Note that Sah and Stiglitz $(1985,1986)$ make use of exogenously given probabilities to express this ability.

${ }^{39}$ Following Kaniovski's (2008) terminology it could be said that the problem solving power of an organization (collective problem solving power) is based upon two ingredients: the power resulting from the positions in the organizational architecture (positional power) and the power rooted in the individual actors which are placed into these positions (individual problem solving power).
} 
where each of them satisfies all but one of the axioms of the corresponding theorem. For showing logical independence we focus on the three theorems above concerning the case $\epsilon=1$ as similar examples can be found for $\epsilon=0$ and $\epsilon \in[0,1]$.

Let us begin by demonstrating the logical independence of the three axioms in Theorem 4.4 by making use of three alternative power scores.

A1. The zero-power score $f^{z e r o}$ is an extreme that considers every actor to be powerless in any situation by assigning zero power to every actor $i$ in any hierarchy $(N, D)$, i.e., $f_{i}^{\text {zero }}(N, D)=0$ for all $i \in N$. This power score satisfies truncation and the bound property. It does not satisfy normalization.

A2. The dictator-power score $f^{\text {dictator }}$ assumes that the proposal goes to the top actor $\left\{i_{0}\right\}$ without any effect of the other actors, and the top actor fully decides about acceptance or rejection, i.e., $f_{i_{0}}^{\text {dictator }}(N, D)=2$ and $f_{i}^{\text {dictator }}(N, D)=0$ for all $i \in N \backslash\left\{i_{0}\right\}$. This power score satisfies normalization and truncation. It does not satisfy the bound property.

In order to define a third power score let $\widetilde{K}(D) \subseteq K(D)$ be the set of bottom actors having the shortest path to the top actor, i.e., $i \in \widetilde{K}(D)$ if and only if $\left|\widehat{S}_{j}^{-1}(D)\right| \geq\left|\widehat{S}_{i}^{-1}(D)\right|$ for all $j \in K(D)$.

A3. The short-power score $f^{\text {short }}$ makes use of the idea behind the power score $\eta^{1}$, but only applies it to those bottom actors who have the shortest path to the top actor, i.e., $f_{i}^{\text {short }}(N, D)=\frac{1}{|\widetilde{K}(D)|} \sum_{\substack{(T, D(T)) \in \mathcal{T}_{D}^{i_{0}} \\ i \in T, b(T) \in \widetilde{K}(D)}} \eta_{i}^{1}(T, D(T))$, where $b(T)$ is the bottom actor in the truncated hierarchy $T$, i.e., $b(T) \in T$ and $S_{D}(b(T))=\emptyset .{ }^{40}$ This power score satisfies normalization and the bound property. It does not satisfy truncation.

Next, we show the logical independence of the four axioms in Theorem 4.9 by making use of the three power scores above and a further fourth power score.

B1. The zero-power score $f^{z e r o}$ defined under A1 satisfies symmetry, independence of outside organization, and the successor-sum property. As mentioned before, it does not satisfy normalization.

B2. The dictator-power score $f^{\text {dictator }}$ defined under A2 satisfies normalization, symmetry, and independence of outside organization. It does not satisfy the successor-sum property.

B3. The short-power score $f^{\text {short }}$ defined under A3 satisfies normalization, symmetry, and the successor-sum property. It does not satisfy independence of outside organization.

In order to define the fourth power score let $l_{D}$ be the lowest labeled bottom actor in $(N, D)$, i.e., $k \geq l_{D}$ for all $k \in K(D)$.

B4. The low-power score $f^{l}$ considers the truncated hierarchy with the lowest labeled bottom actor as bottom actor only, i.e., $f_{i}^{l}(N, D)=\eta_{i}\left(\widehat{S}^{-1}\left(l_{D}\right) \cup\left\{l_{D}\right\}, D\left(\widehat{S}^{-1}\left(l_{D}\right) \cup\left\{l_{D}\right\}\right)\right)$ if $i \in \widehat{S}^{-1}\left(l_{D}\right) \cup\left\{l_{D}\right\}$ and $f_{i}^{l}(N, D)=0$ otherwise. This power score satisfies normalization, independence of outside organization, and the successor-sum property. It does not satisfy symmetry.

Finally, we show logical independence of the three axioms in Theorem 5.3 by making use of three alternative power measures.

\footnotetext{
${ }^{40}$ Note that we take the sum over the empty set to be equal to zero.
} 
C1. The zero-power measure is defined as its corresponding power score $f^{z e r o}$ under A1. ${ }^{41}$ This power measure satisfies strong independence of outside organization and regularity. It does not satisfy normalization.

C2. The dictator-power measure is defined as its corresponding power score $f$ dictator under A2. This power measure satisfies normalization and strong independence of outside organization. It does not satisfy regularity.

The third power measure makes use of our definition of an office (see Sect. 5.1) where the set of all offices $\{S(j)\}_{j \in N \backslash K(D)}$ is a partition of $N \backslash\left\{i_{0}\right\}$.

C3. The constant-office-power measure $f^{\text {office }}$ assigns to every office the same power of 2, i.e., it is defined by $f_{i_{0}}^{\text {office }}(N, D)=2$ and for every $j \in N \backslash K(D), f_{i}^{\text {office }}(N, D)=\frac{2}{|S(j)|}$ for all $i \in S(j)$. This power measure satisfies normalization and regularity. It does not satisfy strong independence of outside organization.

\section{References}

Aumann, R. J., \& Drèze, J. H. (1974). Cooperative games with coalition structures. International Journal of Game Theory, 3, 217-237.

Banzhaf, J. F. (1965). Weighted voting doesn't work: a mathematical analysis. Rutgers Law Review, 19, 317343.

Berg, S., \& Paroush, J. (1998). Collective decision making in hierarchies. Mathematical Social Sciences, 35 , 233-244.

Bolton, P., \& Dewatripont, M. (1994). The firm as a communication network. Quarterly Journal of Economics, 109, 809-839.

Braham, M. (2008). Social power and social causation: towards a formal synthesis. In M. Braham \& F. Steffen (Eds.), Power, freedom, and voting (pp. 1-21). Berlin: Springer.

Braham, M., \& Holler, M. J. (2008). Distributing causal responsibility in collectivities. In T. Boylan \& R. Gekker (Eds.), Economics, rational choice, and normative philosophy London: Routledge.

Braham, M., \& Steffen, F. (2002). Voting power and games with abstention. In M. J. Holler et al. (Ed.), Power and fairness (pp. 333-348). Tubingen: Mohr Siebeck.

Brams, S. (1968). Measuring the concentration of power in political systems. American Political Science Review, 62, 461-475.

van den Brink, R. (1994). Relational power in hierarchical organizations. PhD dissertation, Tilburg University.

van den Brink, R. (1997). An axiomatization of the disjunctive permission value for games with a permission structure. International Journal of Game Theory, 26, 27-43.

van den Brink, R. (1999). An axiomatization of the conjunctive permission value for games with a hierarchical permission structure. In H. de Swart (Ed.), Logic, game theory and social choice (pp. 125-139). Tilburg University Press.

van den Brink, R. (2001). An axiomatization of the Shapley value using a fairness property. International Journal of Game Theory, 30, 309-319.

van den Brink, R. (2002). Apex games as relational power measures for directed graphs. Social Choice and Welfare, 19, 845-867.

van den Brink, R. (2008). Vertical wage differences in hierarchically structured firms. Social Choice and Welfare, 30, 225-243.

van den Brink, R., \& Gilles, R. P. (1996). Axiomatizations of the conjunctive permission value for games with permission structures. Games and Economic Behavior, 12, 113-126.

van den Brink, R., \& Gilles, R. P. (2000). Measuring domination in directed networks. Social Networks, 22, 1141-1157.

van den Brink, R., \& Steffen, F. (2008). Positional power in hierarchies. In M. Braham, F. Steffen (Eds.), Power, freedom, and voting (pp. 57-81). Berlin: Springer.

\footnotetext{
${ }^{41}$ Note that this is the only power measure that is 'consistent' with $f$ zero as a power score in the sense that a measure is obtained from a normalized score.
} 
Chandler, A. D. (1966). In Strategy and structure New York: Doubleday.

Chwe, M. S.-Y. (2000). Communication and coordination in social networks. Review of Economic Studies, $67,1-16$.

Copeland, A. H. (1951). A reasonable social welfare function. Mimeo, Seminar on Applications of Mathematics to Social. Sciences, University of Michigan.

Daudi, P. (1986). Power in the organization. Basil: Blackwell.

Felsenthal, D. S., \& Machover, M. (1996). Alternative forms of the Shapley value and the Shapley-Shubik index. Public Choice, 87, 315-318.

Felsenthal, D. S., \& Machover, M. (1997). Ternary voting games. International Journal of Game Theory, 26, 335-351.

Felsenthal, D. S., \& Machover, M. (1998). The measurement of voting power. Cheltenham Glos: Edward Elgar.

Felsenthal, D. S., \& Machover, M. (2004). Analysis of QM rules in the draft constitution for Europe proposed by the European Convention, 2003. Social Choice and Welfare, 23, 1-20.

Felsenthal, D. S., Leech, D., List, C., \& Machover, M. (2003). In defence of voting power analysis: responses to Albert. European Union Politics, 4, 473-497.

Fischer, A. J. (1999). The probability of being decisive. Public Choice, 101, 267-283.

Gilles, R. P., \& Owen, G. (1994). Cooperative games and disjunctive permission structures. Department of. Economics, Virginia Polytechnic Institute and State University, Blacksburg, Virginia.

Gilles, R. P., Owen, G., \& van den Brink, R. (1992). Games with permission structures: the conjunctive approach. International Journal of Game Theory, 20, 277-293.

Grofman, B., \& Owen, G. (1982). A game theoretic approach to measuring degree of centrality in social networks. Social Networks, 4, 213-224.

Harré, R. (1970). Powers. British Journal of the Philosophy of Science, 21, 81-101.

Herings, P. J. J., van der Laan, G., \& Talman, A. J. J. (2005). The positional power of nodes in digraphs. Social Choice and Welfare, 24, 439-454.

Hu, X., \& Shapley, L. S. (2003). On authority distributions in organisations: controls. Games and Economic Behavior, 45, 153-170.

Johnston, P., \& Gill, J. (1993). Management control and organizational behaviour. London: Paul Chapman.

Kaniovski, S. (2008). Straffin meets condorcet: what can a voting power theorist learn from a Jury theorist? Homo Oeconomicus, 25, 1-22.

Laruelle, A., \& Valenciano, F. (2008). Voting and collective decision-making. Cambridge: Cambridge University Press.

Leech, D. (2002). Designing the voting system for the council of the European Union. Public Choice, 113, 437-464.

Mackenzie, K. D. (1976). A theory of group structures, vol. I: Basic theory. New York: Gordon \& Breach.

Martin, J. (1998). Organizational behaviour. International Thompson Business Press.

Matthews, K., \& Thompson, J. (2008). The economics of banking (2nd edn.). New York: Wiley.

Mizruchi, M. S., \& Potts, B. B. (1998). Centrality and power revisited: actor success in group decision making. Social Networks, 20, 353-387.

Morgenstern, O. (1951). Prolegomena to a theory of organization. Rand Research Memorandum RM-5438PR.

Morriss, P. (1987/2002). Power: a philosophical analysis. Manchester: Manchester University Press.

Napel, S., \& Widgrén, M. (2006). The inter-institutional distribution of power in EU codecision. Social Choice and Welfare, 27, 129-154.

Packel, E. W., \& Deegan, J. (1980). An axiomated family of power indices for simple n-person games. Public Choice, 35, 229-239.

Radner, R. (1992). Hierarchy: the economics of managing. Journal of Economic Literature, 30, 1382-1415.

Russett, B. M. (1968). Probalism and the number of units: measuring influence concentration. American Political Science Review, 62, 476-480.

Sah, R. K., \& Stiglitz, J. E. (1985). The theory of economic organizations. AEA Papers and Proceedings, 75, 292-297.

Sah, R. K., \& Stiglitz, J. E. (1986). The architecture of economic systems: hierarchies and polyarchies. American Economic Review, 76, 716-727.

Shapley, L. S. (1962). Simple games: an outline of the descriptive theory. Behavioral Science, 7, 59-66.

Shapley, L. S., \& Palamara, J. R. (2000a). Control games and organizations. UCLA Working Paper 795.

Shapley, L. S., \& Palamara, J. R. (2000b). Simple games and authority structure. UCLA Working Paper 796.

Shapley, L. S., \& Shubik, M. (1954). A method for evaluating the distribution of power in a committee system. American Political Science Review, 48, 787-792.

Shubik, M. (1962). Incentives, decentralized control, the assignment of joint costs and internal pricing. Management Science, 8, 325-343. 
Simon, H. A. (1962). The architecture of complexity. Proceedings of the American Philosophical Society, $106,467-482$.

Smith, C. G., \& Tannenbaum, A. S. (1963). Organizational control structure: a comparative analysis. Human Relations 16, 299-316.

Steffen, F. (2002). Essays in the theory of voting power. PhD Dissertation, University of Hamburg.

Straffin, P.D. Jr. (1977). Homogeneity, independence, and power indices. Public Choice 30, 107-118.

Straffin, P. D. Jr. (1978). Probability models for power indices. In P. C. Ordeshook (Ed.), Game theory and political science (pp. 477-510). New York: New York University Press.

Sutter, M. (2000). Flexible integration, EMU and relative voting power in the EU. Public Choice, 104, 41-62.

Tannenbaum, A. S. (1961). Control and effectiveness in a voluntary organization. American Journal of Sociology, 67, 33-46.

Tannenbaum, A. S., \& Kahn, R. L. (1957). Organizational control structure Human Relations, 10, 127-140.

Williamson, O. E. (1967). Hierarchical control and optimum firm size. Journal of Political Economy, 75, 123-138. 